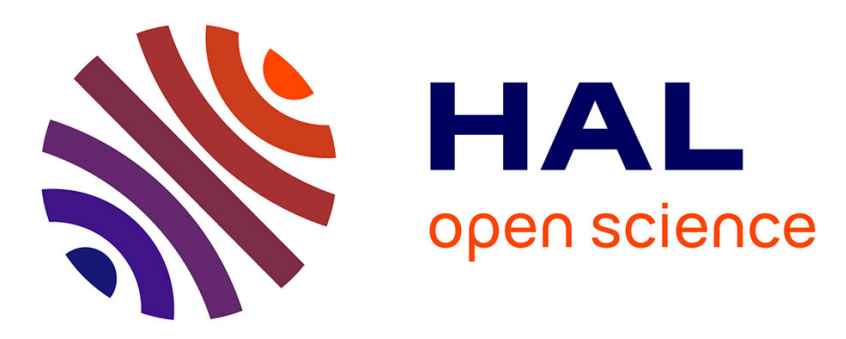

\title{
Feasibility of laser-induced plasma spectroscopy for measurements of equivalence ratio in high-pressure conditions
}

\author{
Laurent Zimmer, Seiji Yoshida
}

\section{- To cite this version:}

Laurent Zimmer, Seiji Yoshida. Feasibility of laser-induced plasma spectroscopy for measurements of equivalence ratio in high-pressure conditions. Experiments in Fluids, 2012, 52 (4), pp.891-904. 10.1007/s00348-011-1151-x . hal-00688379

\section{HAL Id: hal-00688379 \\ https://hal.science/hal-00688379}

Submitted on 17 Apr 2012

HAL is a multi-disciplinary open access archive for the deposit and dissemination of scientific research documents, whether they are published or not. The documents may come from teaching and research institutions in France or abroad, or from public or private research centers.
L'archive ouverte pluridisciplinaire HAL, est destinée au dépôt et à la diffusion de documents scientifiques de niveau recherche, publiés ou non, émanant des établissements d'enseignement et de recherche français ou étrangers, des laboratoires publics ou privés. 


\title{
Feasibility of Laser Induced Plasma Spectroscopy for measurements of equivalence ratio in high-pressure conditions
}

\author{
Laurent Zimmer • Seiji Yoshida
}

Received: date / Accepted: date

\begin{abstract}
In this paper, experimental results obtained with Laser Induced Plasma Spectroscopy to retrieve local compositions are presented for an ambient pressure up to $5.0 \mathrm{MPa}$ in a still cell. Well controlled mixtures of gases are introduced and plasma is obtained with the fundamental emission of a pulsed Nd:YAG laser. Simultaneously, plasma shape and spectrally resolved data are taken with a temporal resolution down to 2 ns. First, the temporal evolutions of a high-pressure nitrogen plasma are analyzed as function of spark energy. It is shown that plasma changes orientation from an elongated shape parallel to the laser line to a perpendicular one in a very short time. Results are reported for both spatial and spectral variations. Afterwards, the effects of increased carbon concentration are discussed in both shape and spectra. It is seen that strong intensity due to the atomic carbon emissions appear for the high-pressure case. From those experiments, calibration strategies are proposed to get equivalence ratio under high pressure conditions with a ratio of carbon versus nitrogen and oxygen. The delay between plasma and measurements is set to $2000 \mathrm{~ns}$ and the signal is integrated for $5000 \mathrm{~ns}$, so as to yield a good signal to noise ratio and a good sensitivity of the technique to changes in mixture fraction. Calibration curves are reported for equivalence ratio up to 1.00 and for pressure from 1.0 to $5.0 \mathrm{MPa}$. It is shown that typical uncertainties are limited to $7.5 \%$ regardless the equivalence ratio in a single shot approach using a spectral fit procedure, whereas it accounts to two times more in a more classical peak ratio approach. Increasing the pressure tends to increase the precision as lower pressure had higher uncertainties.
\end{abstract}

L. Zimmer

CNRS, UPR 288 Laboratoire d'Energétique Moléculaire et Macroscopique, Combustion (EM2C) Grande Voie des Vignes, 92295 Châtenay-Malabry, France

and

Ecole Centrale Paris, Grande Voie des Vignes, 92295 Châtenay-Malabry, France

Tel.: (+33) 141131066

Fax: (+33) 147028035

E-mail: laurent.zimmer@em2c.ecp.fr

S. Yoshida

Japan Aerospace Exploration Agency

7-44-1 Jindaiji-Higashi, 182-8522 Chofu JAPAN 
Keywords Plasma spectroscopy $\cdot$ Laser induced plasma $\cdot$ High-pressure $\cdot$ Equivalence ratio measurements

\section{Introduction}

Measurements of local composition are of great interest for combustion diagnostics. Whereas different strategies may exist for atmospheric conditions, an increase in pressure tends to induce some difficulties. Using gas sampling techniques requires to have sealed working conditions while keeping the possibility to use traversing systems to have multiple points diagnostics. Alternative techniques to the gas sampling using laser techniques have been reported. One may apply a tracer LIF approach, like reviewed in Schulz and Sick (2005) to relate the intensity of a specific molecular fluorescence to the actual fuel to air ratio. This requires the precise knowledge of the amount of the naturally present tracers with respect to overall mass of the fuel or to have a precise control of the injection of the seeded tracers. However, some cautions have to be put when applying this strategies to higher pressures, as reported by Devillers et al. (2009) who studied toluene fluorescence for pressures up to 2.0 MPa. Other approaches, based on different molecules, like vapor Kerosene (Orain et al. (2009)) proved to work for pressures around 2.2 MPa. However, no discussion on single shot uncertainties were presented. Alternatives to tracer LIF may be absorption approaches, based on $\mathrm{C}-\mathrm{H}$ stretching vibration for instance (Hanson (2011)). This kind of approach is leading generally to line of sight measurements, which may not reveal some inhomogeneities in the process. But this kind of technique provides a good overview of the combustion chemistry. Alternative techniques like Raman and Rayleigh may be possible even at high pressures for gaseous fuel but are not possible choices when dealing with liquid fuel, due to much lower threshold in energy for breakdown to occur. Some results were presented with hydrogen for pressures up to 30 bar, but the single shot uncertainty was estimated to be of the order of $\pm 10 \%$ (Kojima and Nguyen, 2004). Due to higher density, maximum fluence before gaseous breakdown tends to decrease; spark's occurrence increases. This is further amplified when using liquid fuel, due to the presence of droplets. One may take advantage of that phenomena to use the spectra from the plasma in order to determine the local concentration of fuel versus air. This technique is called Laser Induced Plasma Spectroscopy and was first introduced in Schmieder (1981) for combustion applications and since that used in many different cases (see for instance Phuoc and White (2002), Sturm and Noll (2003),Stavropoulos et al. (2005)). Different strategies may be applied when measuring at atmospheric pressure. The most commonly used consists in using the ratio of hydrogen $\left(\mathrm{H}_{\alpha}\right.$ at $656 \mathrm{~nm}$ or $\mathrm{H}_{\beta}$ at $486 \mathrm{~nm}$ ) over an atomic emission of oxygen (usually $777 \mathrm{~nm}$ ) or nitrogen (around $744 \mathrm{~nm}$ ) like discussed in Phuoc and White (2002). Other strategies have been proposed where the atomic emission of carbon at $711 \mathrm{~nm}$ is used to depict the concentration of fuel whereas oxidizer is determined using the triplet of nitrogen emission around $742-746 \mathrm{~nm}$ (see Ferioli and Buckley (2006)). Traditional approaches use spark energy for classification; meaning that the same spark energy is assumed when doing experiments and calibration. An alternative approach has been presented by Zimmer and Tachibana (2007) in which the actual excitation of the plasma is used when applying the calibration curves. This consists in using different emission lines of the same atom (typically hydrogen) to determine a mean temperature of the plasma and then using appropriate calibration curves to link the different atoms to their original concentration. Single shot uncer- 
tainty for mixtures around equivalence ratio of 0.5 was found to be around $7 \%$ in a spark energy classification scheme and dropped to $3 \%$ for the excitation scheme. It was also shown that ambient temperature had no effect on the calibration, nor a change in the laser source. Further applications to different gaseous mixtures (hydrogen-air and helium-air) as well as some theoretical aspects have been recently proposed by Zimmer et al. (2007). It was shown that N/O can also be used for getting an insight in the excitation of the plasma, even though less sensitive when compared to the original hydrogen ratio. Whereas those measurements were done in atmospheric conditions, the present paper deals with cases under higher pressures, from 0.1 to $5.0 \mathrm{MPa}$ for different mixture of gases. Experiments of LIPS applied to high-pressure cases were already reported but for solid targets (Arp et al., 2004). It was for instance shown that typical radii of plasma were smaller when increasing the pressure by a factor a cube root of the ratio of the pressure. Another issue that was demonstrated is the problems of self-absorption when increasing the pressure. Self-absorption could be detected through the broadening of the emission and the resulting decrease of intensity in the middle of the emission profile for a specific line. The limited effects of broadening was also shown by Vors et al. (2008) who studied carbon emissions in nitrogen gases. The pressures tested were ranging up to $8.0 \mathrm{MPa}$. The carbon emission considered was the $247.86 \mathrm{~nm}$ and only time integrated data (over $20 \mu \mathrm{s}$ ) were presented. The lifetime of the plasma was shown to decrease with pressure. However, in none of the previous investigations, a detailed description of the dynamics of those high pressure cases was described. Furthermore, no quantitative evaluation of the recorded spectra were proposed. The relative emission of the carbon was considered by integrating the signal around the emission peak and neighbourtgh emissions exceeding tenth of peak height. Interestingly, no self-absorption was noticed with the selected carbon emission. The purpose of the present paper is to investigate the dynamics of high-pressure laser induced plasma and to propose strategies to get quantitative data (in the present case in terms of equivalence ratio) even at 5.0 MPa. The second section of the article presents the details of the experimental setup. In the third section, the spatial evolution of the plasma as function of plasma energy, gaseous composition and pressures is presented and discussed. The fourth section deals with the spectroscopic data while the fifth shows the two different strategies for getting quantitative data. Finally conclusions are drawn for those high pressure cases and typical applications of the technique are suggested.

\section{Presentation of the setup and processing techniques}

To conduct high-pressure tests, a specific test-cell has been built. It is equipped with three optical accesses, two for the laser beam and one perpendicular for imaging purpose. The high-pressure test cell has a length of $198 \mathrm{~mm}$ and is equipped with a fan and a vacuum pump. Before each test, vacuum is made down to $0.001 \mathrm{MPa}$. To reach the desired mixture, four different gases are used: $\mathrm{N}_{2}, \mathrm{O}_{2}, \mathrm{CH}_{4}$ and $\mathrm{CO}_{2}$. To achieve a homogeneous mixture, gases are introduced according to their partial pressure (lowest being first introduced) and final temperature is around 293K. Thermocouple as well as pressure measurements are performed while making the measurements to ensure that the conditions stay stable within the experimental campaign. Equivalence ratio is computed on a basis of atomic ratio between carbon and oxygen, assuming the fuel may be represented by $\mathrm{C}_{n} \mathrm{H}_{2 n+2}$. If applied to practical flames, the technique is not 
sensitive to the actual molecules composing the fuel as all molecules will be dissociated into atoms. The fundamental emission from a Nd:YAG (Quanta Ray Spectra Physics, $1064 \mathrm{~nm}$ ) is used to create a plasma. Its beam is focussed through a series of concave $(\mathrm{f}=-30 \mathrm{~mm})$ and convex lenses $(\mathrm{f}=120 \mathrm{~mm}$ and $\mathrm{f}=250 \mathrm{~mm})$ into the test cell without damaging the windows. Two energy meters are used to measure incident and outgoing laser energy (Ophir Head). To calibrate the energy meter, vacuum is created inside the cell so that plasma can not be achieved, even when passing through the different optics with a high energy. A convex lens $(\mathrm{f}=120 \mathrm{~mm})$ is used after the cell to converge the laser beam to the energy meter. A linear correlation is obtained and is used to measure the spark energy. It is assumed that the difference detected on the two energy meters comes only from the creation of the spark and therefore the spark energy is attributed this difference. A mirror for $1064 \mathrm{~nm}$ is used between the laser and the cell to allow emission analysis in a backward configuration (see Figure 1). This ensures that even for fluctuations in the position of the plasma along the axis of the laser beam, spectra are always measured completely and with the same efficiency with the spectrometer.

The signal from the plasma is provided to a spectrometer through an optical fiber. This spectrometer is equipped with a grating of 150 lines $/ \mathrm{mm}$ and its slit is set at $50 \mu \mathrm{m}$ and connected to an ICCD (Andor, 1024x1024) used in full vertical binning mode. The single shot measurements can encompass a spectral band of $320 \mathrm{~nm}$. To achieve fully spectrally resolved measurements, two different series are taken. In the first series, the center wavelength is set at $470 \mathrm{~nm}$, while in the second one, it is set at $740 \mathrm{~nm}$. Finally, the region accessible with this setup is therefore $300-910 \mathrm{~nm}$. To monitor both plasma shape and position, an ICCD (Roper Scientific, 1024x1024) equipped with an objective of $105 \mathrm{~mm}$ focal length is used. No filters are used so as to measure complete natural emission of the plasma. Integration time for this camera was always adjusted so as to avoid any damages to the detector even with the high photon flux. This will allow discussing the initial growth and afterwards cooling process as function of spark energy, gaseous composition and pressure. Time resolved measurements are obtained by varying the time delay between plasma and measurements and by adjusting the integration time. This is done by synchronizing all measurements with a delay generator (BNC555). For each conditions, a series of 200 shots are taken. Gains for both ICCD are adjusted so as to not saturate the signal while keeping a good signal to noise ratio. Exact initial timing is set for each Q-switch settings on both ICCD's. This has to be done so as to capture the initial creation of the plasma as delays may change due to changes in the laser pulse. Typical temporal analysis may take 45 minutes for a complete description of the statistical changes of the plasma with respect to time. Over the entire duration of a time series, pressure and temperature is monitored in the cell and no fluctuations are observed.

\section{Spatial evolution of laser induced plasma under different pressures and gaseous composition}

The first measurements deal with the geometrical evolution of the laser induced plasma as function of time, pressure, gaseous composition and spark energy. 


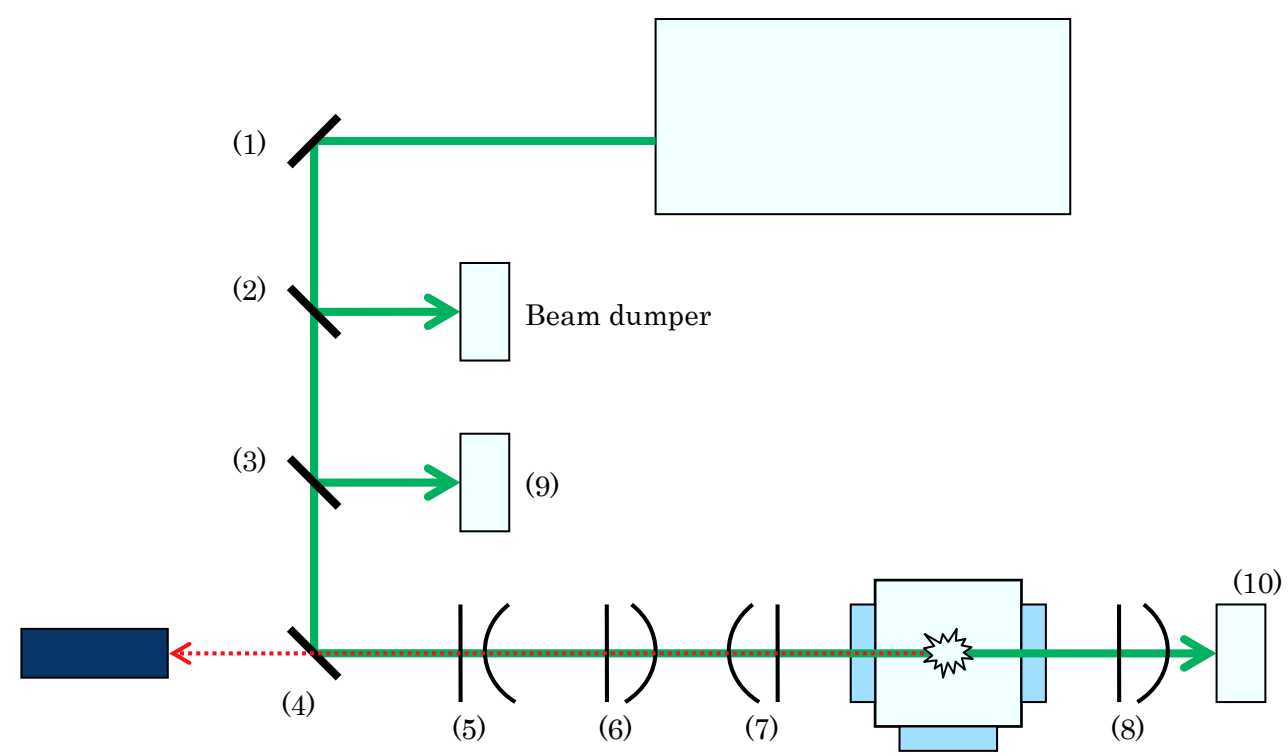
(1) Mirror for $1064 \mathrm{~nm}$,
Sigma koki, TFMHP-50C08-1064
(2) Mirror for $532 \mathrm{~nm}$,
CVI Laser, $\quad$ Y2-2037-45-UNP
(3) Beam sampler,
Sigma koki, BS4-30C03-10-550
(4) Dichroic mirror,
Sigma koki, YHS-50C08-1064
(5) Concave lens, $f=-30 \mathrm{~mm}$,
Sigma koki, SLB-20-30N
(6) Convex lens, $f=120 \mathrm{~mm}$,
Sigma koki, SLB-50.8-120P
(7) Convex lens, $f=250 \mathrm{~mm}$,
Sigma koki, SLB-100-250P
(8) Convex lens, $f=120 \mathrm{~mm}$,
Sigma koki, SLB-50.8-120P
(9) Energy meter 1,
OPHIR, PE50-BB-DIF
(10) Energy meter 2,
OPHIR, PE50-BB-DIF

Fig. 1 Detailed sketch of the optical layout. 
3.1 Influence of pulse energy on the evolution of the shape

To quantify the effects of pressure and spark energy on the shape of the laser induced plasma, several conditions are used. Keeping the laser energy constant, different plasma are created as function of gaseous pressure. The list of conditions are summarized in Table 1. Temporally resolved measurements are averaged over more than 150 shots to discuss the influence of those different parameters on the actual shape of the plasma and its temporal evolution.

\begin{tabular}{|c|c|c|c|c|c|}
\hline Pressure & $0.1 \mathrm{MPa}$ & $0.5 \mathrm{MPa}$ & $1.0 \mathrm{MPa}$ & $2.5 \mathrm{MPa}$ & $5.0 \mathrm{MPa}$ \\
\hline Laser Pulse $(060 \mathrm{~mJ})$ & - & - & - & 18 & 27 \\
\hline Laser Pulse $(106 \mathrm{~mJ})$ & 58 & 65 & 66 & 68 & 77 \\
\hline Laser Pulse $(160 \mathrm{~mJ})$ & 111 & 109 & 112 & 115 & 125 \\
\hline Laser Pulse $(195 \mathrm{~mJ})$ & 142 & 145 & 147 & 148 & 160 \\
\hline
\end{tabular}

Table 1 Plasma energy $(\mathrm{mJ})$ as function of laser pulse energy and gaseous pressure

One can see that for very low pulse energy, it is not possible to create a plasma for pressures below 2.5 $\mathrm{MPa}$ with the selected optical components. One has to recall that a series of three lenses were used to focus the plasma. This is mainly to avoid creation of plasma on the optical access. Another interesting feature is that the plasma energy is not changing for pressures encompassed between 0.5 and $2.5 \mathrm{MPa}$, whereas cases for the highest pressure always present a plasma energy about $10 \%$ higher than cases at $2.5 \mathrm{MPa}$ and that only one case at $0.1 \mathrm{MPa}$ leads to a plasma energy $10 \%$ lower (for a pulse energy of $106 \mathrm{~mJ}$ ). One can already conclude that potential differences may not be attributed to differences in plasma energy.

To retrieve a mean temporal evolution, single images are analyzed individually. It is checked that plasma energy falls within the desired boundaries, $\pm 1 \mathrm{~mJ}$ around the mean value reported in Table 1 . Typical images are integrated over the entire wavelength and therefore are very bright all over the time. The aperture of the objective is set to 32 and gain remains equal to zero all over the tests as no interferential filter is used. Typical examples of an instantaneous image for a low energy and for a high energy plasma case are shown in Figure 2 and Figure 3. Those images have been obtained for a delay of $1000 \mathrm{~ns}$ and an integration time of $100 \mathrm{~ns}$. One can see some differences in the instantaneous shape of plasma, even though ambient pressure and temporal settings are identical. The intensity has been normalized between 0 and 1 and only levels from 0.1 are represented, as intensity below 0.1 is considered as being noise. The low energy case has an aspect ratio relatively large, whereas the plasma becomes already more spherical for the spark of $160 \mathrm{~mJ}$ case. In both cases, the laser beam comes from the left. The exact position of the center of the plasma changes with the energy. In the present case, for clarity reason, the mean position of the plasma at the earliest delay is set to $0 \mathrm{~mm}$ in both directions. This allows a discussion on the evolution of the length and height as well as the changes in the center as function of time. One can notice a region with a quite uniform emission above 0.8 which also has a different shape as function of plasma energy. To better analyze the shape of the plasma as function of the various parameters, the mean evolution is computed. 


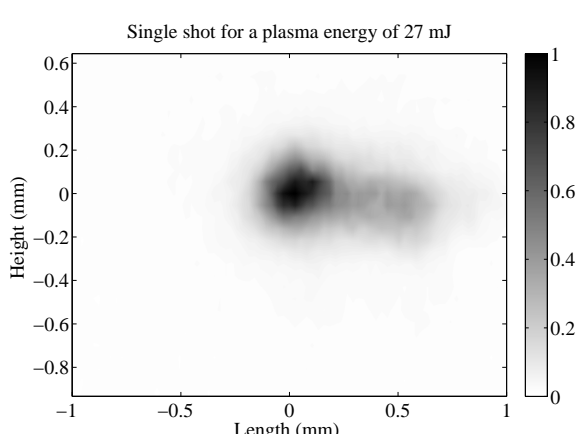

Fig. 2 Typical plasma shape for $\mathrm{N}_{2}$ at $5.0 \mathrm{MPa}$, a delay of $1000 \mathrm{~ns}$ and an integration of $100 \mathrm{~ns}$ for a spark of $27 \mathrm{~mJ}$.

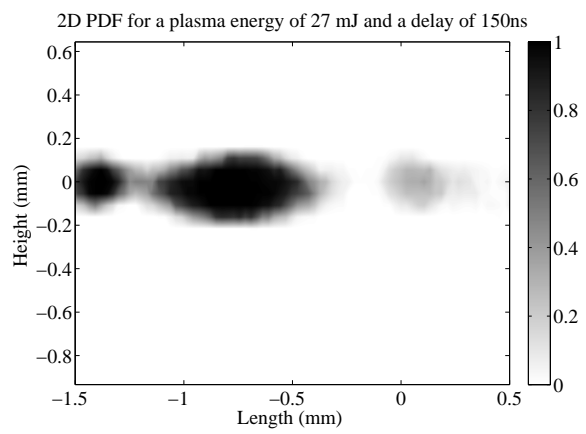

Fig. 4 Typical 2D PDF of the plasma shape for $\mathrm{N}_{2}$ at $5.0 \mathrm{MPa}$ and a delay of $150 \mathrm{~ns}$ for a spark of $27 \mathrm{~mJ}$.

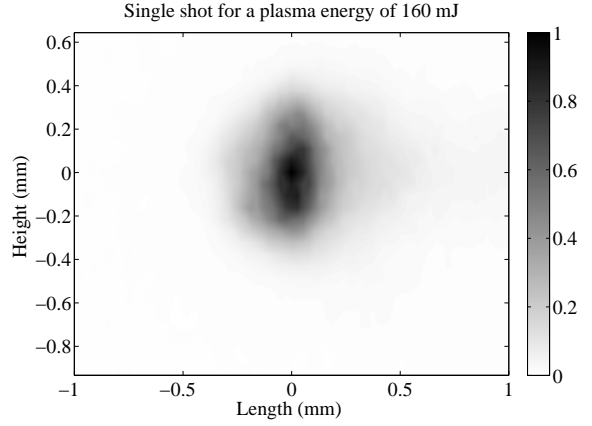

Fig. 3 Typical plasma shape for $\mathrm{N}_{2}$ at $5.0 \mathrm{MPa}$, a delay of $1000 \mathrm{~ns}$ and an integration of $100 \mathrm{~ns}$ for a spark of $160 \mathrm{~mJ}$.

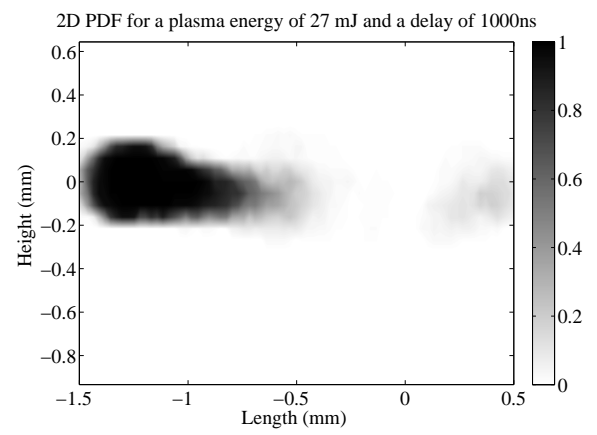

Fig. 5 Typical 2D PDF of the plasma shape for $\mathrm{N}_{2}$ at $5.0 \mathrm{MPa}$ and a delay of $1000 \mathrm{~ns}$ for a spark of $27 \mathrm{~mJ}$.

An intensity threshold is first used to define the boundary of the plasma on each single shot. The threshold is a simple binarization process as background noise remains limited for the different cases under study. It is taken as being the intensity corresponding to $25 \%$ of the maximum intensity, as providing the best signal to noise. The length and height of the plasma are determined and typical fluctuations in position and aspect ratio are discussed. The two dimensional probability density function (2D PDF) to find plasma at given location is also computed based on the analyses of all shots within one series. This gives an overview of the reproducibility of the plasma, while keeping all other parameters constant.

Two examples of such PDF distribution are presented for the case of a plasma of $27 \mathrm{~mJ}$ in Figure 4 for earlier delays and in Figure 5 for later delays. The $\mathrm{x}$-axis and $\mathrm{y}$-axis are set at zero for the highest probability at the earliest time (which is in the present case obtained at a delay of $0 \mathrm{~ns}$ ).

One can notice that the two shapes are very different. Whereas initial delays tend to offer an elongated plasma with several discontinuities (suggesting multiple plasma), later delays show a more compact shape. Furthermore, one can notice a small shift in the central position towards the direction of the laser (as laser comes from left to right). This shift in time is enhanced with an increase of plasma energy. This has some 
direct consequences on the LIPS technique as the probe volume is not only changing in size and shape but also in space. Therefore, LIPS technique without an ICCD camera to measure the location of the formation of the plasma may not be very accurate as far as position of the probe volume is concerned. To allow a better analysis, the width and height are then defined as the sizes corresponding to a probability higher than $10 \%$ on those two dimensional probability density function. This threshold offers the best compromise between accuracy and resolution. A lower threshold tends to include more noise and a higher clearly limits the measurement volume. Furthermore, with a low threshold, the multiple plasma situation of Figure 4 would lead to an overestimated size of the plasma. For this case, the length is considered to be the maximum length of the continuous plasma, ranging from -1.5 to about $-0.5 \mathrm{~mm}$ for the case presented in Figure 4.

Results are presented in Figure 6 where both dimensions of $\mathrm{X}$ (along the laser axis, hence referenced as width) and Y (perpendicular to the laser, hence referenced as height) are represented as function of time delay with respect to the time of creation of the plasma. The width and the height are computed as being the longest distance between two points of the plasma. This is required as in some cases two distinct regions are detected, separated by a small region having lower probability. One can first clearly identify the initial effects of plasma energy, as the width of the plasma increases with the plasma energy. The width is about two times longer when creating a plasma of $160 \mathrm{~mJ}$ compared to a spark of $27 \mathrm{~mJ}$. One has a longer ionized region. The influence on the height is less important. This is due to the fact that the initial height is fixed by the optical setup.

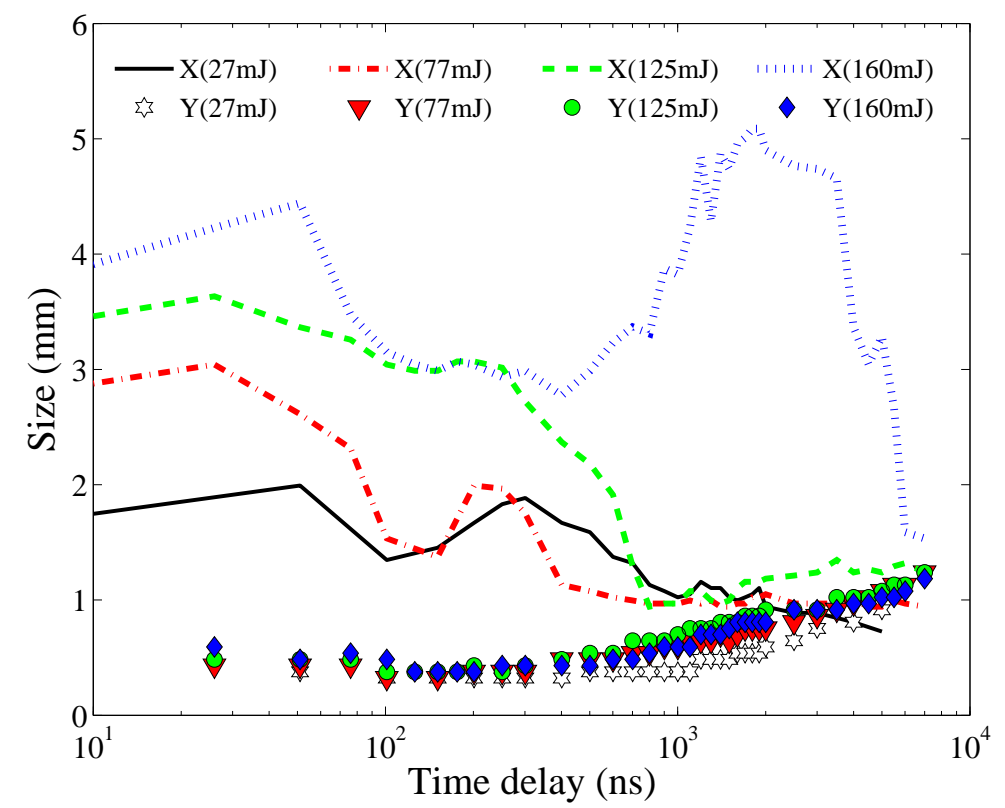

Fig. 6 Temporal evolution of both dimensions for $\mathrm{N}_{2}$ at 5.0 MPa for different spark energies. 
After an initial growth, the plasma tends to contract itself for all energies (for time delays around $100 \mathrm{~ns}$ ). This contraction is not due to an expansion in the other direction as shown by the measurements of the height of the plasma. After this contraction, there is a phase of expansion in the transverse direction. The plasma also changes in shape, from an elongated in the horizontal direction, through a circular shape to an elongated plasma in the vertical direction (a height bigger than the width) for the low energy cases. In this evolution, one can also notice the influence of plasma energy. Low energies tend to delay the vertical growth and limit its expansion, as shown by the results obtained for a plasma energy around $27 \mathrm{~mJ}$. On the other side, little effects are noticed for plasma energies higher than $77 \mathrm{~mJ}$. The three cases have a vertical expansion relatively similar, while having strong differences in the horizontal direction. Through those results, it is clear that the horizontal direction is always influenced by the plasma energy. Higher the energy, longer will be the plasma for delays up to 7000 ns. However, in the vertical axis, no differences are noticed. It is also important to understand that pressure tends to concentrate the time axis, as typical results obtained for atmospheric pressures would not show such a vertical expansion for the plasma emission within the delays investigated here. It is important to recall here that no flow is imposed to the gas. The third lobe generation mechanism (see Bradley et al. (2004) for instance) is however not seen with the plasma direct emission. Those results have some direct consequences for LIPS measurements. If the spatial resolution has to be restricted to the smallest size possible, delays around 100 to 500 ns should be privileged. On the other hand, if one can accept a bigger probe volume, it seems more interesting to take measurements for delays higher than $1500 \mathrm{~ns}$ to have a more spherical probe volume. A compromise will also include spectral constraints as one may expect more ionized atoms at early stages. Assuming a cylindrical shape for the plasma and a third dimension identical to the height, it is possible to compute the volume of the plasma in terms of cubic millimeter. Results are represented in Figure 7 for the four different energies at the highest pressure $(5.0 \mathrm{MPa})$. One can see that at most, the volume is around $2 \mathrm{~mm}^{3}$ for this high pressure case. Between time delays lower than $1000 \mathrm{~ns}$, it is limited to values lower than $1 \mathrm{~mm}^{3}$. An increase in energy creates a bigger probe volume. Another interesting feature of low energy plasma is the relatively constant volume over the time. Actual probe volume may be a bit bigger if one takes long integration time due to the shift of the plasma towards the laser source as function of time, as discussed earlier.

\subsection{Influence of pressure on the evolution of the shape}

Conducting similar analyses for different gaseous pressures, the evolution for the lowest energy of laser pulse that leads to a creation of plasma for all pressures is investigated. Results are obtained for both the longitudinal evolution (along the laser path, labelled length) as well as for the transverse evolution (labelled height). Results are summarized in terms of probe volume, assuming a cylindrical symmetry and presented in Figure 8.

For lower pressures, the plasma tends to expand in both directions up to delays of the order of $2000 \mathrm{~ns}$. For this delay, the typical length is of the order of $6 \mathrm{~mm}$ whereas the height reaches $3.25 \mathrm{~mm}$. For this case, the LIPS measurement is integrated over this entire volume, which, assuming cylindrical revolution along the axis of the laser results in a probe volume of $15 \mathrm{~mm}^{3}$ at most. Higher pressures lead to initially smaller probe volume. For pressures below 2.5 MPa, a maximum volume is reached for delays around $1000 \mathrm{~ns}$ whereas the volume increases for the two highest pressure cases 


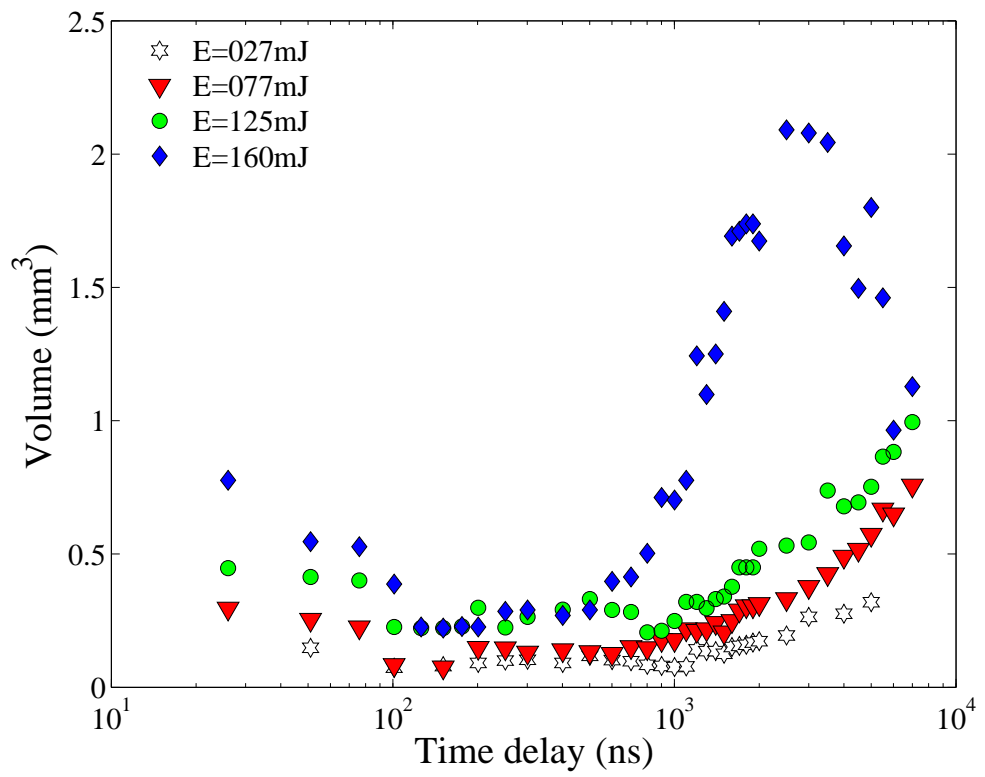

Fig. 7 Temporal evolution of the probe volume for $\mathrm{N}_{2}$ at 5.0 MPa for different spark energies.

investigated here even for delays around $2000 \mathrm{~ns}$. It is also interesting to recall that the lowest pressure case was leading to a plasma energy of only $58 \mathrm{~mJ}$ whereas the highest pressure had a mean plasma energy of $77 \mathrm{~mJ}$. This clearly shows that pressure is a key factor for the volume and as already noticed by Vors et al. (2008), the plasma is smaller for a higher pressure. The tendency discussed in Arp et al. (2004) for which the volume was a linear function of pressure is not found in the present investigation. One of the reason is that in Arp et al. (2004), plasma was created on a solid target whereas in the present case, it is free to evolve along the three directions.

\subsection{Effect of gaseous composition}

To discuss the temporal evolution of plasma shape as function of gaseous composition, a procedure similar to the one presented before is used. The four different gaseous compositions are listed in Table 2 and presented as function of their partial pressures. The partial pressure of nitrogen is always adjusted so as to recreate the proper ratio of air with respect to oxygen. Oxygen is not inserted in the cell in presence of fuel, so as to avoid ignition of the mixture. The global equivalence ratio $(\phi(\mathrm{C}))$ can be determined based on the ratio of carbon to oxygen and assuming a fuel of typical composition $\mathrm{C}_{n} \mathrm{H}_{2 n+2}$.

The temporal evolution of both length and width for the different mixtures is represented in Figure 9 for a spark energy of $27 \mathrm{~mJ}$ and an ambiant pressure of 5.0 MPa.

One can distinguish two regions. For small delays (less than $500 \mathrm{~ns}$ ), there is little influence of gaseous composition on the plasma expansion. Both dimensions are similar regardless the presence of oxygen, carbon or hydrogen and the resulting probe volume is limited to about $0.1 \mathrm{~mm}^{3}$. However, for delays higher than $500 \mathrm{~ns}$, the length of the 


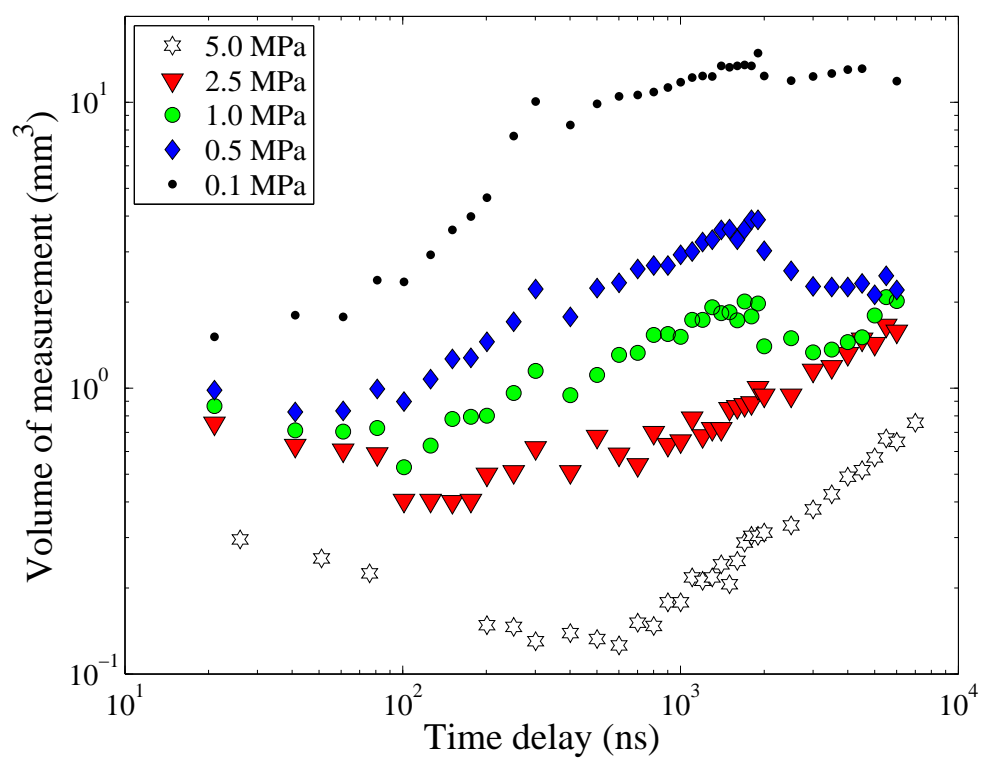

Fig. 8 Temporal evolution of the probe volume as function of pressure for a laser pulse of $106 \mathrm{~mJ}$.

\begin{tabular}{|c|c|c|c|c|c|}
\hline Label & $\mathrm{N}_{2}$ & $\mathrm{O}_{2}$ & $\mathrm{CO}_{2}$ & $\mathrm{CH}_{4}$ & $\phi(\mathrm{C})$ \\
\hline $\mathrm{N}_{2}$ & 1.00 & 0.00 & 0.00 & 0.00 & 0.00 \\
\hline $\mathrm{N}_{2} \mathrm{O}_{2}$ & 0.79 & 0.21 & 0.00 & 0.00 & 0.00 \\
\hline $\mathrm{N}_{2} \mathrm{CO}_{2}$ & 0.79 & 0.00 & 0.21 & 0.00 & 1.50 \\
\hline $\mathrm{N}_{2} \mathrm{CO}_{2} \mathrm{CH}_{4}$ & 0.78 & 0.00 & 0.20 & 0.02 & 1.65 \\
\hline
\end{tabular}

Table 2 Partial pressure of the different gases for the four mixtures investigated at 5.0 MPa.

plasma obtained in presence of carbon clearly increases to reach a maximum of $2.5 \mathrm{~mm}$ for delays around 1500-1700 ns. This is seen for both mixtures containing carbon. In parallel to this, a radial expansion can also be noticed, resulting in an overall increase of the probe volume. At most, there is a factor 5 for the probe volume with and without carbon at delays of 1000 ns. For longer delays, there is little difference induced by carbon concentration and typical probe volume are of the same order for delays after 5000 ns. Again, this emphasizes the need to have a simultaneous measurement of the probe volume has its size is influence not only by pressure and laser energy but also by gaseous composition.

It is interesting to see if this increase in the probe volume is accompanied by an overall increase in all the emissions are more specifically to some emissions only. Therefore, the spectral analysis is proposed in the next section to see if similar effects are detected as increasing carbon content. 


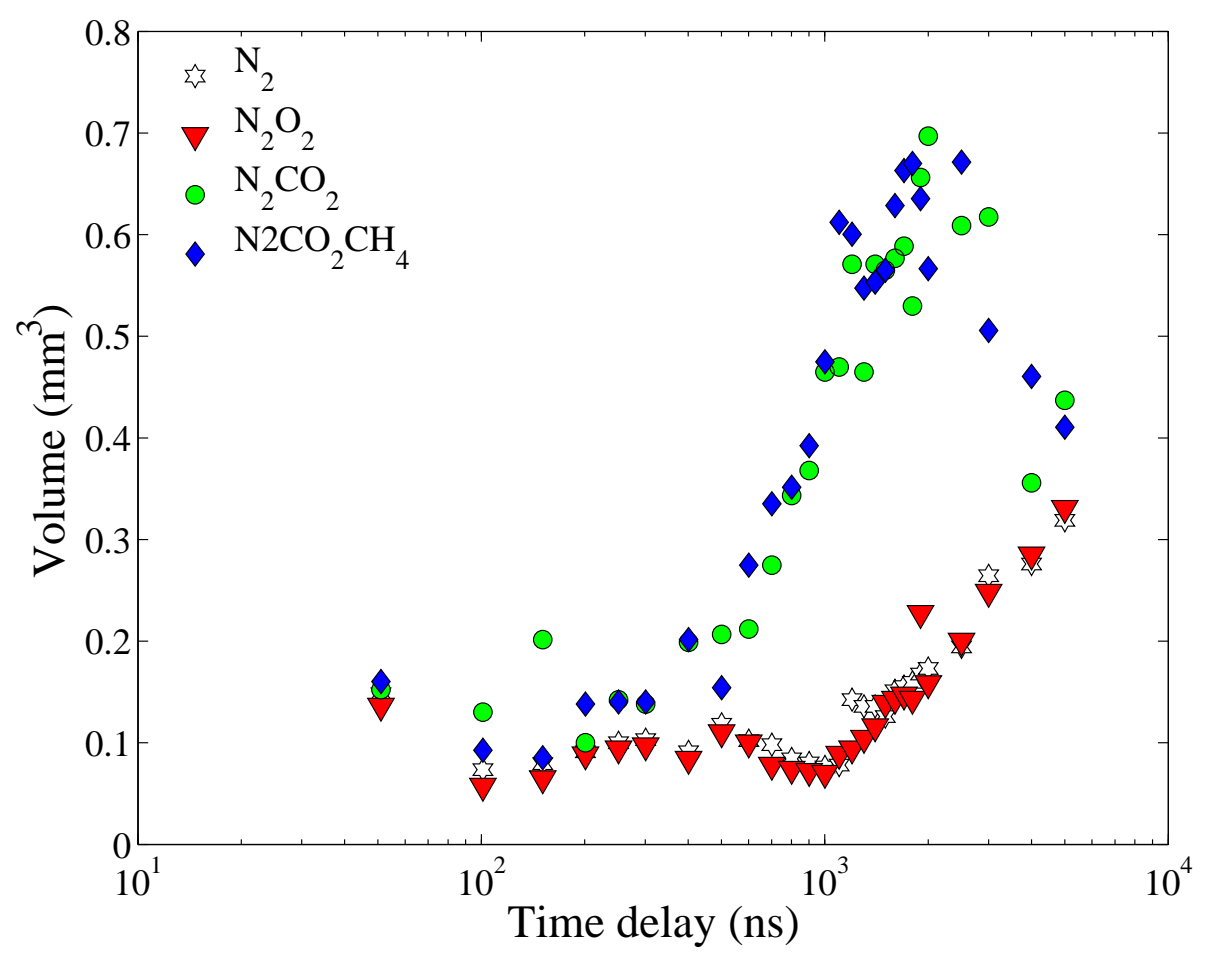

Fig. 9 Temporal evolution of plasma shape for different compositions at 5.0 MPa and a spark energy of $27 \mathrm{~mJ}$.

\section{Spectral measurements}

\subsection{Processing of the spectral data}

To get an absolute evolution at different wavelengths, the quantum efficiency of the system is first determined. This is done by getting the efficiency at some discrete points using well calibrated lamps. To determine the equivalence ratio from spectral data, three different strategies are proposed. The first one is a classical approach in LIPS. One integrates all the intensity under the selected emission lines. This is done by determining the background level at the selected wavelengths assuming it can be linearly interpolated between two neighboring points (one on the left and the other on the right) that have no interference with any emission lines. This takes into account the possible multiple emission like the triplet of nitrogen around 742, 744 and $746 \mathrm{~nm}$. Another strategy consists in fitting the best simulated spectra with the experimental spectra. This strategy is similar to the one presented in Zimmer et al. (2007) where simulations were reported for atmospheric cases. In the present case, simulations should include effects of pressure for the different atomic emissions concerned: nitrogen, oxygen and carbon. Therefore, an alternative strategy is proposed. It is based in first determining experimentally an averaged spectra for a given mixture fraction with specific time delay and integration. Averaged spectra are then used to create a database. All instan- 
taneous spectra are correlated with this database and the best fit can be determined. The actual value of the single shot equivalence ratio may be further interpolated between two averaged spectra assuming a gaussian profile for the correlation and using a three-points scheme. This strategy aims at using all the spectral information available and not only few discrete lines as it would be the case for the classical approach.

The uncertainties will be discussed in the following sections for both air cases and air-dioxide carbon cases. In this section, individual emission lines are discussed following a classical approach to explain each specific atomic emissions, whereas the global fitting will be used in the fifth section when retrieving quantitative concentration from single shot spectra.

4.2 Temporal evolution of the spectral emissions

To discuss the overall evolution, mean spectra at different time delays are taken. Those instantaneous spectra (a typical example being represented in Figure 10) are averaged over each delays. It is extremely difficult to analyze such a single shot as the gate width is limited to $100 \mathrm{~ns}$, but it is possible to distinguish some emissions induced by oxygen around $777 \mathrm{~nm}$ and other emissions around $820 \mathrm{~nm}$, induced by both nitrogen and oxygen.

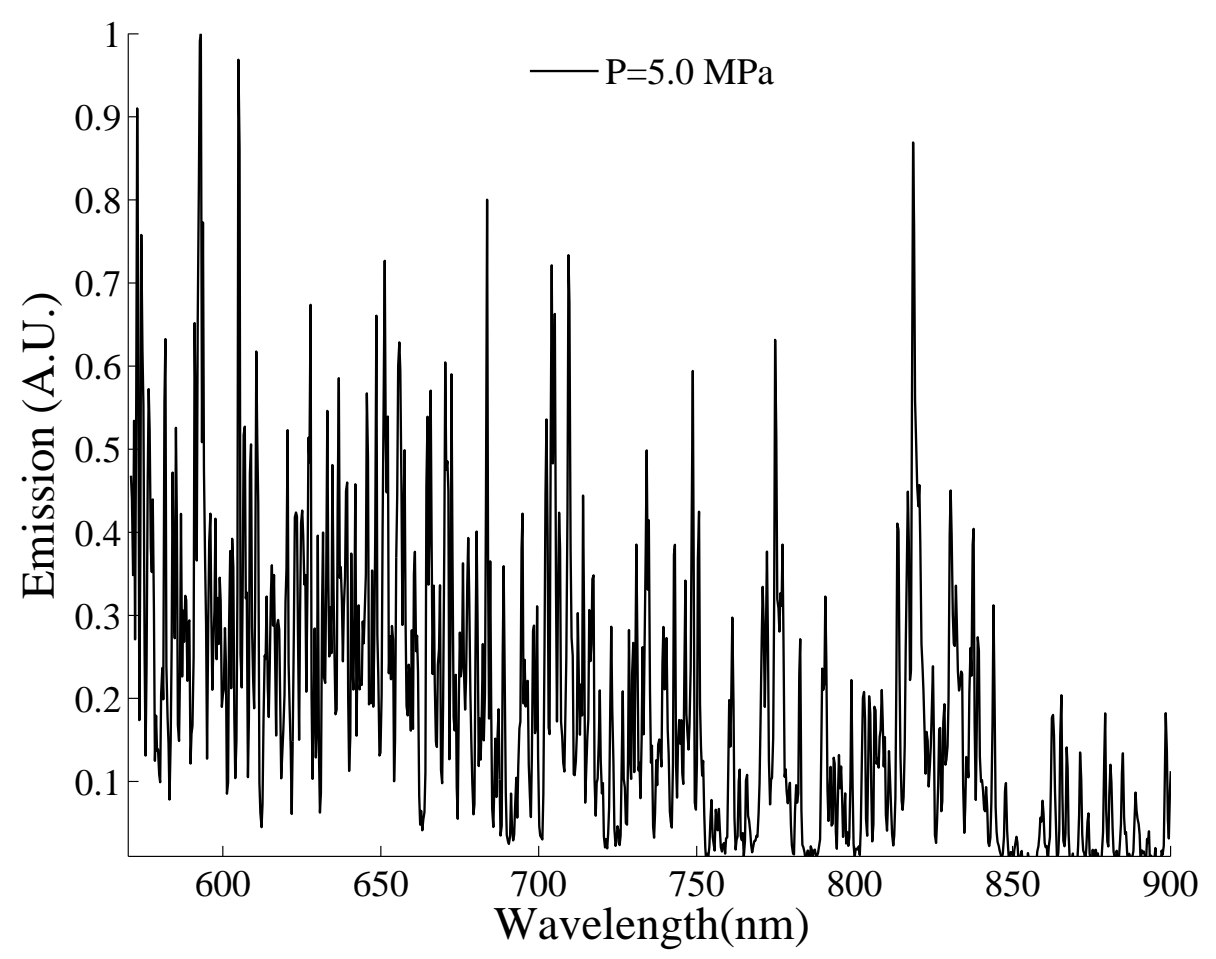

Fig. 10 Typical single shot spectra for a delay of $1500 \mathrm{~ns}$ an integration time of $100 \mathrm{~ns}$ for a plasma energy of $27 \mathrm{~mJ}$ and $\mathrm{N}_{2} \mathrm{CO}_{2}$ mixture at $5.0 \mathrm{MPa}$. 
Then, to describe the typical spectral evolution and to take into account the exponential decrease of the intensity with time, it is decided to plot the relative spectral evolution at each time step. After averaging the spectra and taking into account the quantum efficiency, the averaged spectra are normalized so as to have a maximum of unity. Afterwards, repeating this operation at different time delays and for different gaseous compositions, a relative temporal evolution of spectra can be obtained. As the purpose of the present investigation is to see the feasibility of Laser Induced Plasma Spectroscopy to measure equivalence ratio at high pressure, the relative evolutions of nitrogen-oxygen and nitrogen-carbon dioxide are compared in Figure 11 and 12 for a pressure of 5.0 MPa.

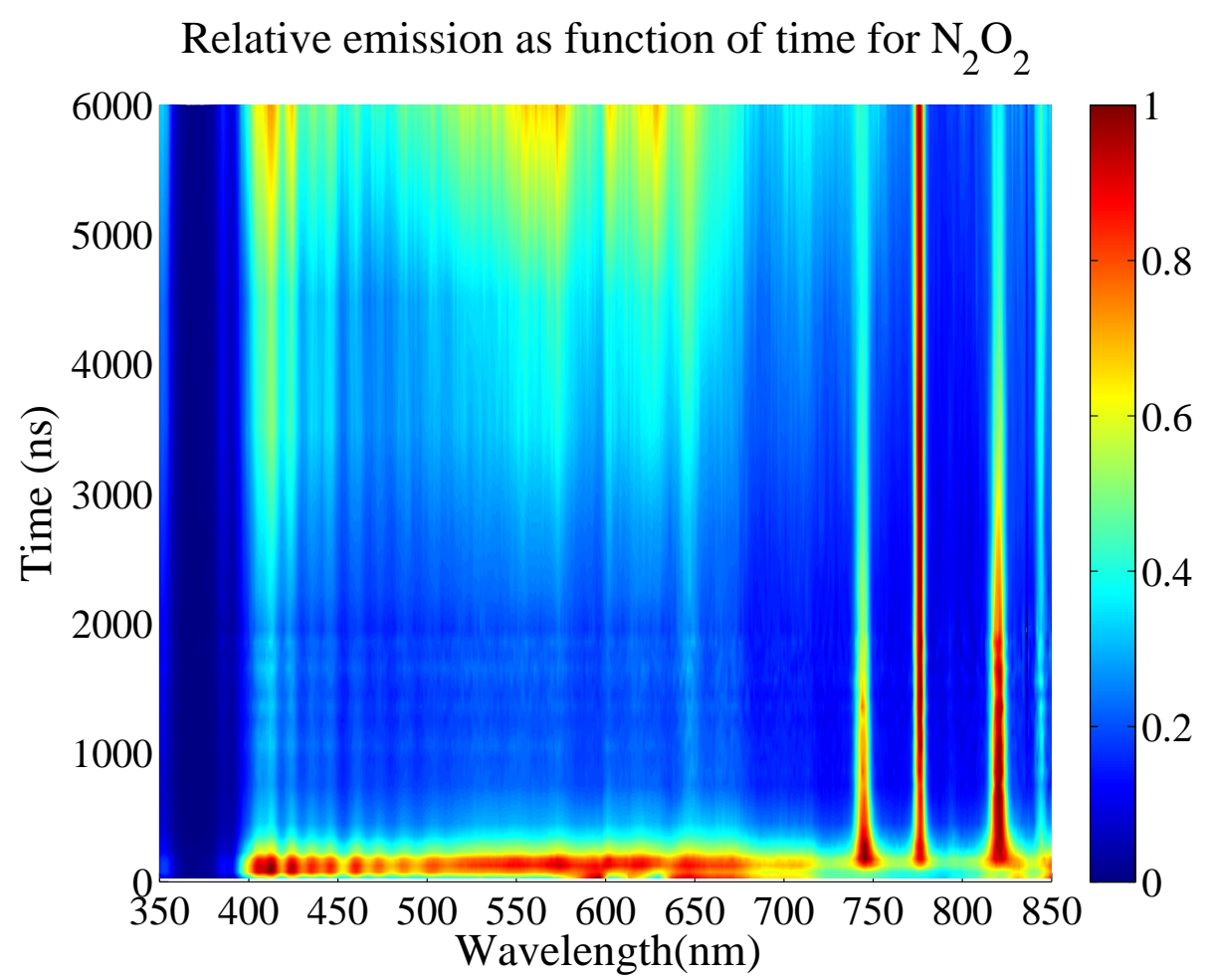

Fig. 11 Temporal evolution of the relative spectra for an energy of $27 \mathrm{~mJ}$, a pressure of 5.0 $\mathrm{MPa}$ and $\mathrm{N}_{2} \mathrm{O}_{2}$ gaseous mixture.

When analyzing first the nitrogen-oxygen case, one can see an initial phase for which a continuum is relatively important. This continuum is mainly induced by some ionic emissions of nitrogen around 400-450 nm. Further delays reveal the emission of atomic lines of both nitrogen and oxygen. It is interesting to notice that the emission of the oxygen remains the most intense emission for delays after $200 \mathrm{~ns}$ up to delays aroud $6000 \mathrm{~ns}$. One has however to recall that the absolute intensity drops exponentially with time. Later delays also reveal different emission regions, even though no clear emission from molecules are visible. The triplet of nitrogen around $742-746 \mathrm{~nm}$ 


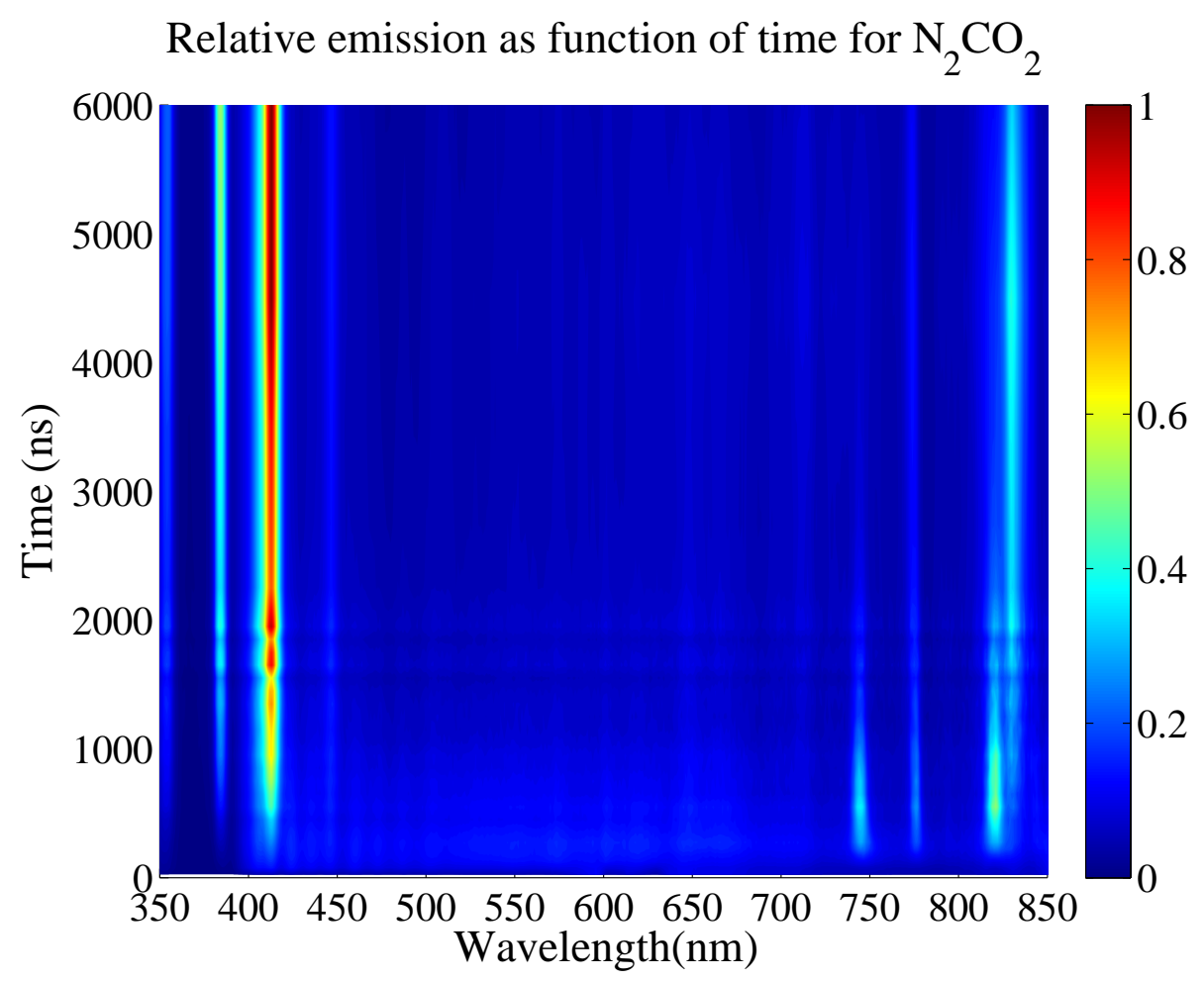

Fig. 12 Temporal evolution of the relative spectra for an energy of $27 \mathrm{~mJ}$, a pressure of $5.0 \mathrm{MPa}$ and $\mathrm{N}_{2} \mathrm{CO}_{2}$ gaseous mixture.

as well as the emission around $820 \mathrm{~nm}$ from oxygen are visible. This graph also reveals that broadening remains limited even at 5.0 MPa and that the spectral width remains constant with time. The accuracy of the present spectral measurements is estimated to be $1 \mathrm{~nm}$. From these analyses, it is possible to conclude that averaged spectra can be used to discuss the relative emissions of nitrogen-oxygen.

Adding carbon (nitrogen- carbon dioxide conditions) reveals a strongly different spectral evolution and shape. Together with the apparition of new specific emissions, a huge difference is seen in the dynamics of the spectra. Whereas the peak around $400 \mathrm{~nm}$ was not predominant for the nitrogen-oxygen case, its strength comes clearly out in the carbonate atmosphere when compared to the emission at $445 \mathrm{~nm}$ for delays bigger than $100 \mathrm{~ns}$. Furthermore, this coincides with an increase of the peak around $385 \mathrm{~nm}$. There are two possible origins for the peak around $385 \mathrm{~nm}$, both being molecular emission either of CN (violet system) or $\mathrm{C}_{2}$ (Deslandres-D'Azambuja system) (see Acquaviva (2004)). The high-pressure environment may tend to ease molecular formation for smaller delays as compared to atmospheric pressure. The spectral accuracy as well as pressure broadening effects make a clear identification of molecular spectroscopy within the present framework impossible. Another clear peak attributed to carbon can be distinguished around $833 \mathrm{~nm}$ compared to the nitrogen-oxygen emission around $820 \mathrm{~nm}$. The peak from carbon is coming from the transition between $61981.82 \mathrm{~cm}^{-1}$ 
and $73975.91 \mathrm{~cm}^{-1}$. The relative strength of carbon increases with time whereas nitrogen and oxygen decreases. It is interesting to note that this peak is a bit delayed compared to the peaks of nitrogen and oxygen. A possible explanation resides in the ionization levels of respectively carbon, oxygen and nitrogen. The ionization energy for carbon being the smallest (11.26 eV compared to $13.61 \mathrm{eV}$ and $14.53 \mathrm{eV}$ ), it may lead to a formation of delayed atomic emissions.

From those considerations, it seems possible to take into account carbon emission as fuel marker. It is interesting to notice that no self-absorption could be shown in the present experiments. One possible reason is the spectral resolution chosen, which may be too limited to see such effects. From this comparative analyses, it seems possible to retrieve concentrations but at delays higher than $800 \mathrm{~ns}$, to have the best signal to noise ratio possible for carbon emission. The ultra-violate emissions of carbon are difficult to use as one needs for a quantitative equivalence ratio measurement a line representing also oxygen (or nitrogen). Given the spectral resolution, it is impossible to encompass in a single shot both emissions around $400 \mathrm{~nm}$ and $780 \mathrm{~nm}$. Therefore, the following analyses are based on the relative evolution of emissions between $700 \mathrm{~nm}$ and $850 \mathrm{~nm}$.

The spectral evolution for different plasma energy is not proposed in the present context as the induced probe volume would far increase the typical probe volume required in treating turbulent combustion situations and only the lowest energy case is presented.

\subsection{Specific evolution of $\mathrm{N}(\mathrm{I}), \mathrm{C}(\mathrm{I})$ and $\mathrm{O}(\mathrm{I})$}

In order to propose calibration curves, the specific temporal evolution of $\mathrm{N}, \mathrm{C}$ and $\mathrm{O}$ are shown for a mixture of $\mathrm{N}_{2} \mathrm{CO}_{2}$. For each emission line, the true quantum efficiency is used together with the temporal gate width in order to compute relative emission independent of temporal integration. For each line, the local background is subtracted. Furthermore, all lines are normalized with respect to the overall maximum of the different lines; which in this case is from the nitrogen-oxygen around $820 \mathrm{~nm}$ at a delay of 275 ns. For carbon atoms, two different lines are considered. The strongest emission is around $833 \mathrm{~nm}$. For this case, the emission between $828 \mathrm{~nm}$ and $838 \mathrm{~nm}$ is taken to represent its emission and the local background is computed on regions from 792 to $797 \mathrm{~nm}$ on the left side and from 852 to $857 \mathrm{~nm}$ on the right side. A second emission line is used around $711 \mathrm{~nm}$, which consists in a triplet between higher state around $69700 \mathrm{~cm}^{-1}$ and lower state around $83700 \mathrm{~cm}^{-1}$. To represent nitrogen emissions, the triplet around $742-746 \mathrm{~nm}$ is used whereas oxygen atoms are only tracked around $777 \mathrm{~nm}$. Finally, the emission around $820 \mathrm{~nm}$ is also checked as coming from a combination of nitrogen-oxygen emission lines.

Results are plotted in Figure 13 in a logarithmic scale for the relative intensity to take into account the exponential decrease of intensity with time.

One can distinguish clearly a different behavior between the atoms of nitrogen and oxygen and the atoms of carbon. N(I) emission is decreasing sharply with time after a peak at $275 \mathrm{~ns}$. There is a little difference between pure nitrogen cases and other compositions, due to the fact that the partial pressure of nitrogen is higher for the pure case. Those typical behavior are also found at smaller pressures. The initial increase is mainly due to a cooling process in which initial ions are retrieving the missing electrons to give raise to atomic emission again. The further decrease is still induced by the 


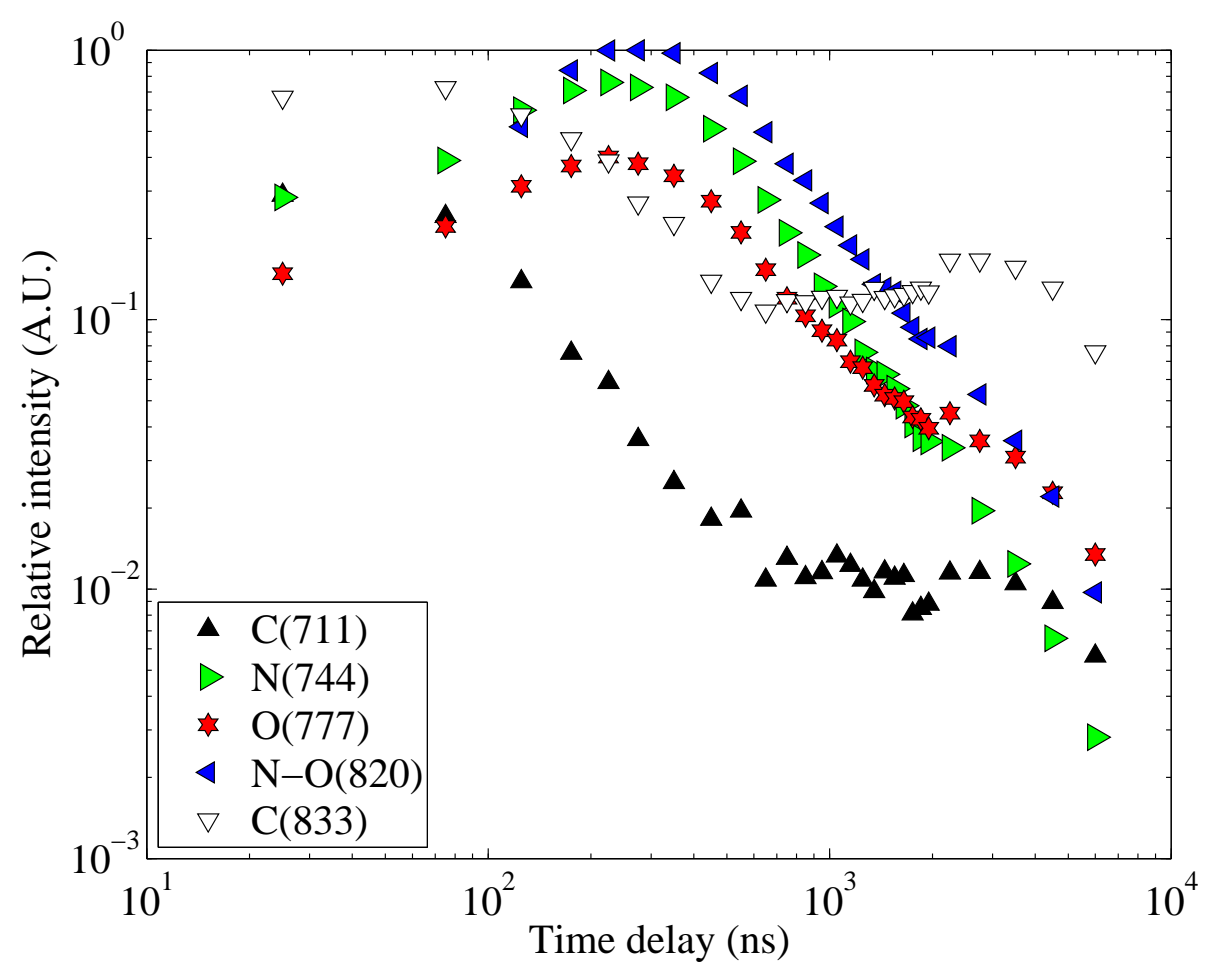

Fig. 13 Temporal evolution of $\mathrm{N}(\mathrm{I}), \mathrm{C}(\mathrm{I})$ and $\mathrm{O}(\mathrm{I})$ for a spark energy of $27 \mathrm{~mJ}$ at a pressure of 5.0 MPa.

cooling process in which the temperature decreasing the probability to have electronic transitions. On the other side, the carbon-linked emissions do present a different temporal behavior. After a constant initial drop, the intensity of the carbon-linked emissions stay almost constant with time, especially at delays between $1000 \mathrm{~ns}$ and $4000 \mathrm{~ns}$. This behavior is independent on the gaseous composition; there is no influence of oxygen on this trend as similar behavior is found for $\mathrm{N}_{2} \mathrm{CO}_{2}$ and $\mathrm{N}_{2} \mathrm{CO}_{2} \mathrm{CH}_{4} \cdot \mathrm{C}(711 \mathrm{~nm})$ emission shows a similar trend as the one shown by $\mathrm{C}(833 \mathrm{~nm})$, with however an emission intensity almost one order of magnitude lower. Therefore, to retrieve local equivalence ratio at pressures up to $5.0 \mathrm{MPa}$, strategies involving carbon emission around $833 \mathrm{~nm}$ and nitrogen-oxygen around $820 \mathrm{~nm}$ may be interesting.

After identifying typical lines to represent the fuel, it is also important to optimize time delays to yield the highest signal to noise ratio possible and check if other ratio may present higher signal to noise ratio or higher sensitivity.

\section{Strategies for equivalence ratio measurements}

With the increase of pressure, carbon emissions are becoming stronger in the short delays as shown in the previous section. Therefore, different calibration strategies may be proposed. A first one is based using the ratio of atomic emission of carbon around 
$833 \mathrm{~nm}$ versus the emisson of nitrogen-oxygen around $820 \mathrm{~nm}$. Another one is to use the ratio of carbon emission around $711 \mathrm{~nm}$ versus the nitrogen emission (from 742 to $746 \mathrm{~nm}$ ), but as presented in the previous section, the relative intensity of this line is weaker than $\mathrm{C}(833)$ and therefore, for simplicity, its evolution is not represented here.

\subsection{Temporal considerations}

An important parameter for calibration is the timing between the plasma creation and the acquisition of the spectra as well as duration of the measurements. For that purpose, the time resolved measurements previously depicted are used to simulate the typical spectral emissions one would obtain when using different integration time. Results are summarized in Figure 14. Five different initial delays have been chosen: 0, 500, 1000, 1500 and $2000 \mathrm{~ns}$ from the initial plasma. As spectra have been taken up to delays of $7000 \mathrm{~ns}$, integration time of $5000 \mathrm{~ns}$ can be obtained for an initial delay of $2000 \mathrm{~ns}$ and an integration time of $7000 \mathrm{~ns}$ if the initial delay is fixed at 0 ns. To obtain such an evolution, a central difference scheme is used. For each delay, the relative $\mathrm{C}(833)$ over $\mathrm{N}(744)$ ratio is computed for different overall integration time. Results clearly show an increase of $\mathrm{C}(833) / \mathrm{N}(744)$ with an increase of the initial delay.

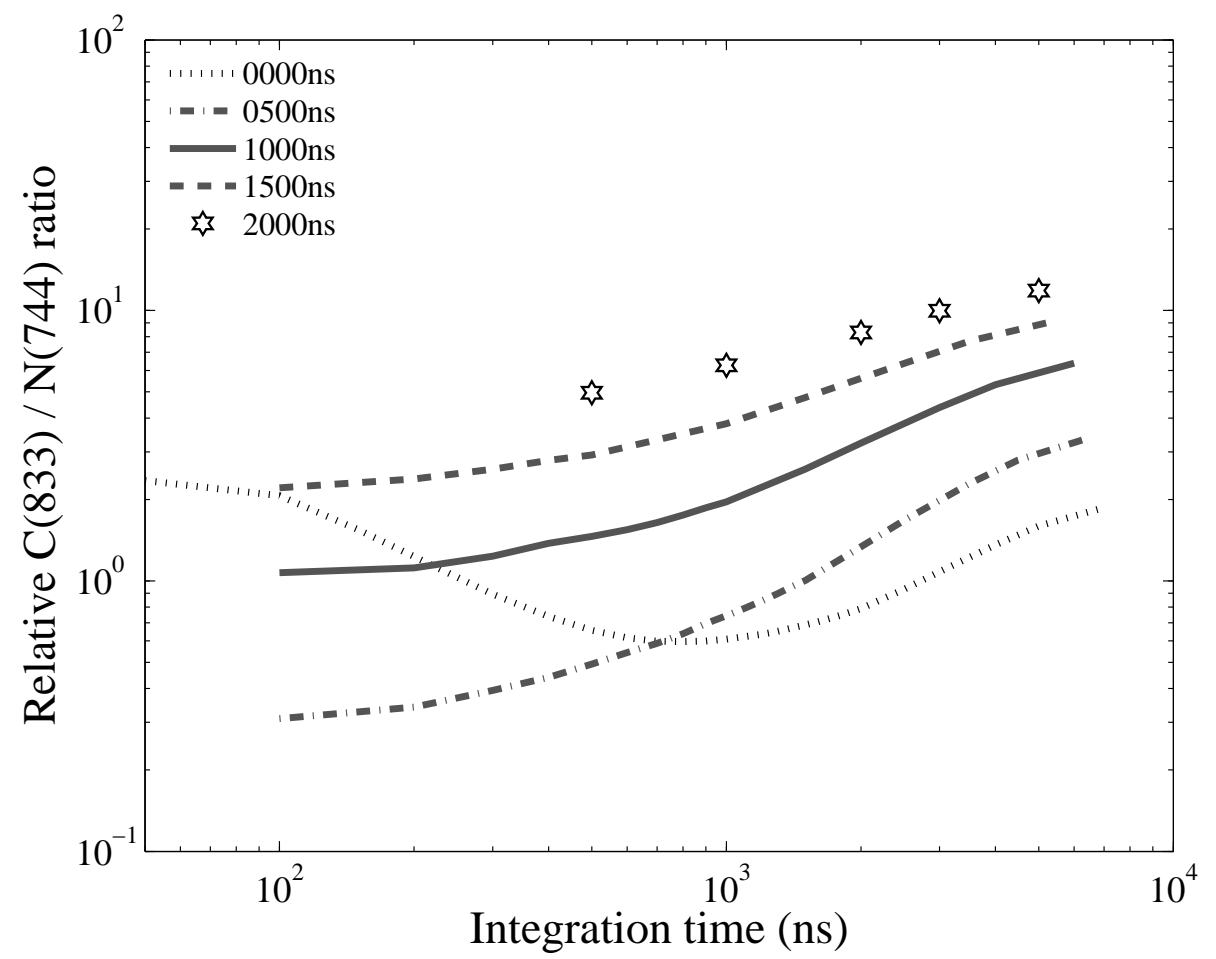

Fig. 14 Temporal evolution of $\mathrm{C}(833)$ over $\mathrm{N}(744)$ as function of initial delay for a spark energy of $27 \mathrm{~mJ}$, for an overall equivalence ratio of 1.50 and a pressure of $5.0 \mathrm{MPa}$ 
It is worth noting that taking the spectra from $0 \mathrm{~ns}$ is clearly not the best choice. Therefore, for such measurements, a gated detector is required to get a good signal. From this plot, it turns out that the higher the initial delay, the best would be the signal for detecting fuel. However, the overall signal is decreasing (as shown in figure 13) and therefore a trade-off has to be found. In the present work, calibrations are reported for delays of $2000 \mathrm{~ns}$ and an integration time of $5000 \mathrm{~ns}$. In the following, the spectra shown are taken with those timing and therefore are not reconstructed from previously used time resolved measurements. A typical single shot spectra taken with those timing is shown in figure 15 for an equivalence ratio of 0.50 . One can recognize on this single shot spectra the different peaks of carbon, nitrogen and oxygen. For each single spectra, the camera noise is first subtracted using spectra obtained without plasma but with identical settings of gain and integration time. Then, the emission due to carbon is computed as being the intensity emitted by the region $833 \pm 5 \mathrm{~nm}$ minus the single shot background (due to broadband emission of plasma) which is computed as being the averaged intensity emitted by the region $800 \pm 5 \mathrm{~nm}$ and $860 \pm 5 \mathrm{~nm}$. The emission of nitrogen is computed in a similar way but with $745 \pm 5 \mathrm{~nm}$ as representing the signal and the averaged of $735 \pm 5 \mathrm{~nm}$ and $763 \pm 5 \mathrm{~nm}$ for the local background. Finally the band $777 \pm 5 \mathrm{~nm}$ is used for the emission of oxygen and the average of $763 \pm 5 \mathrm{~nm}$ and $788 \pm 5 \mathrm{~nm}$ is used for its local background.

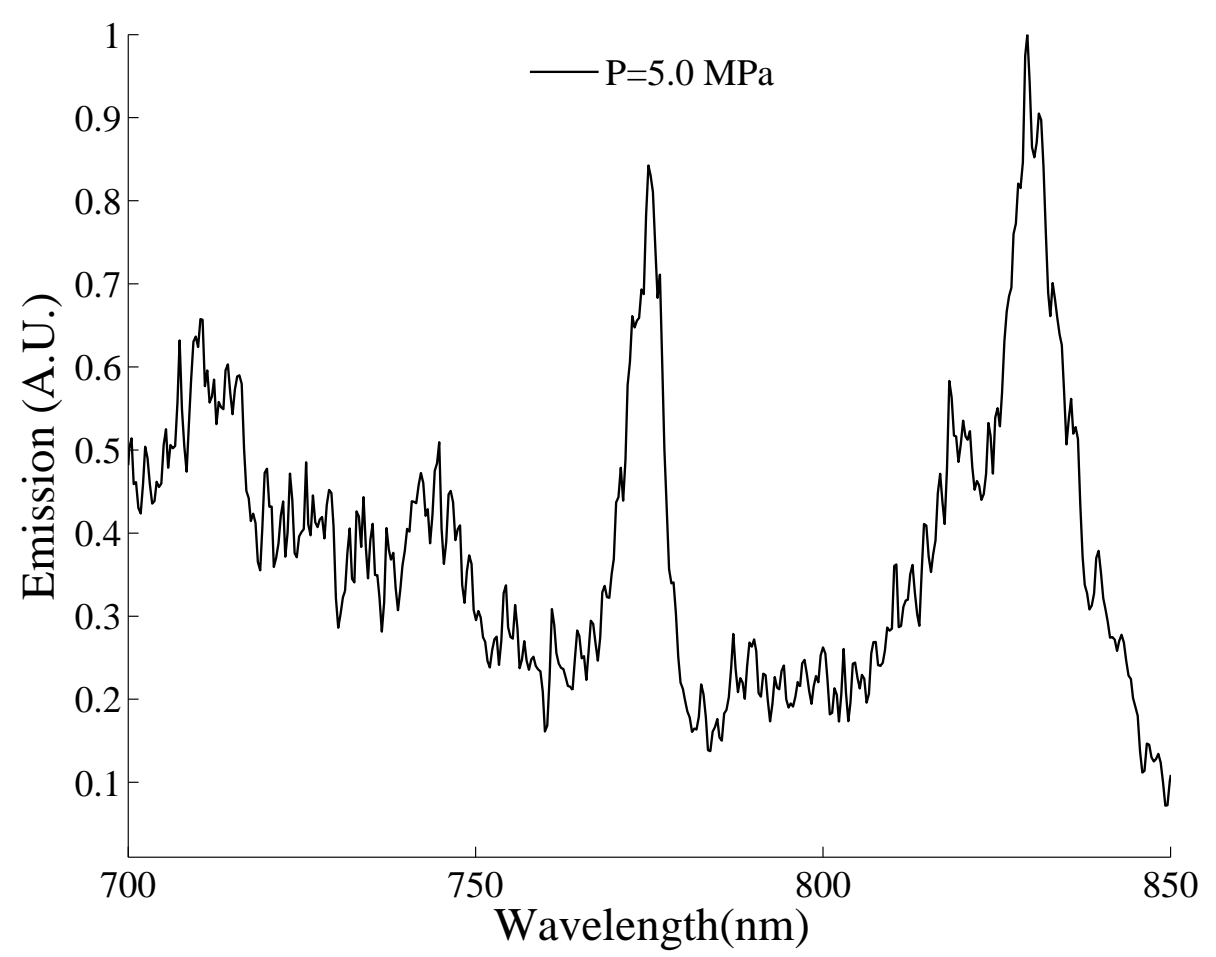

Fig. 15 Single shot spectra for a delay of 2000 ns, an integration time of 5000 ns, a pressure of 5.0 $\mathrm{MPa}$ and an equivalence ratio of 0.50 . 
5.2 Calibration curves

Analyzing more than 150 single shots for each pressure, it is possible to determine calibration curves for the different pressures investigated. Results shown in Figure 16 are compiled over the highest range of pressure investigated for $\mathrm{C}(833)$ emissions to characterize fuel concentration whereas oxidizer is depicted by the N(I) triplet around $742-746 \mathrm{~nm}$ or by the oxygen emission $(777 \mathrm{~nm})$. One can see that a higher pressure leads to a more sensitive ratio as the typical ratio of $\mathrm{C} / \mathrm{N}$ is of the order of 7 at $5.0 \mathrm{MPa}$ whereas it is around 4 at $2.5 \mathrm{MPa}$. The ratio of $\mathrm{C} / \mathrm{O}$ is less sensitive as the slope is about half the slope of $\mathrm{C} / \mathrm{N}$, regardless the gaseous pressures. Data obtained at $1.0 \mathrm{MPa}$ do not show a good behavior as carbon emission around $833 \mathrm{~nm}$ stays weak.

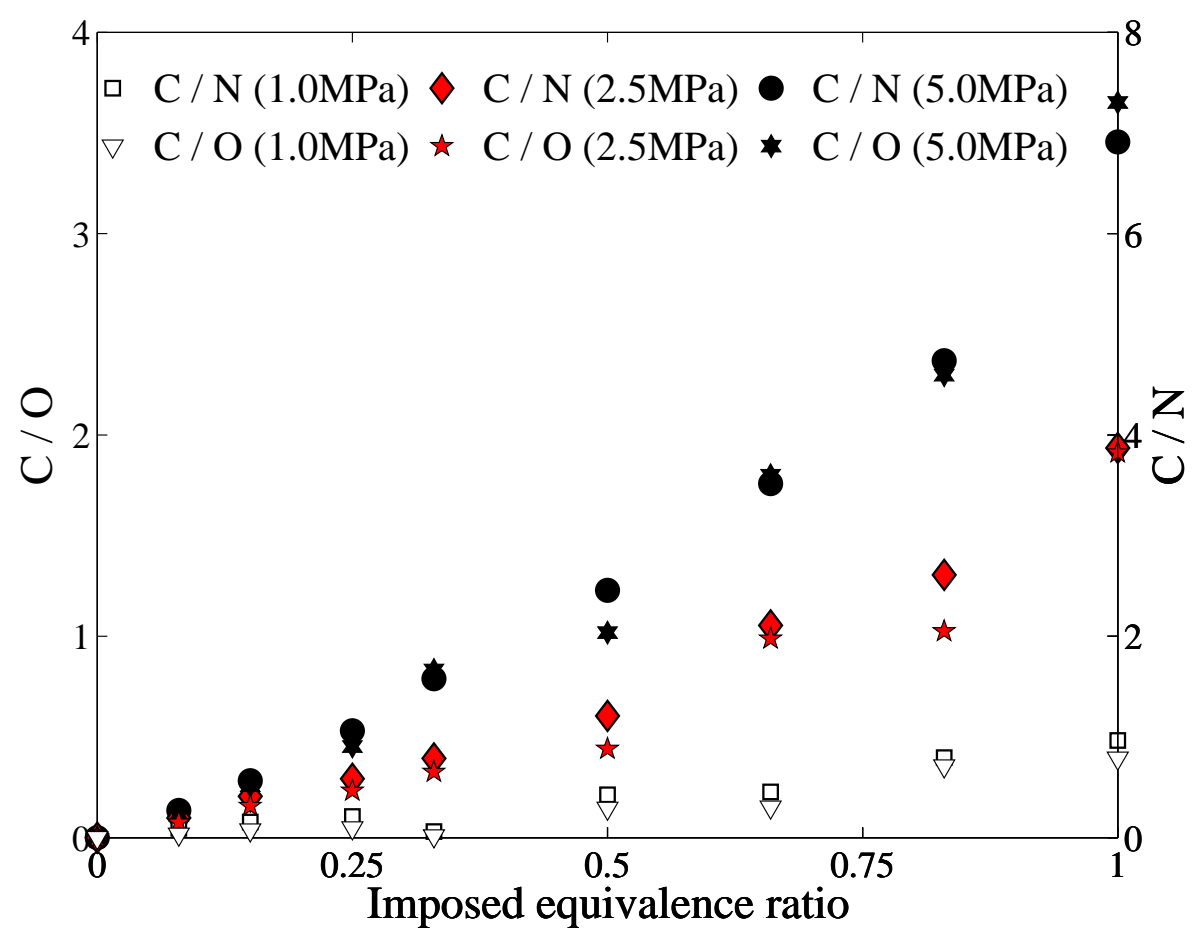

Fig. 16 Calibration curves for different pressures of $\mathrm{C}(833)$ / $\mathrm{N}(744)$ and $\mathrm{C}(833) / \mathrm{O}(777)$ at pressures above 1.0 MPa.

\subsection{Uncertainties}

Treating the 150 shots for each imposed equivalence ratio and using the previously obtained calibration curve, it is possible to compute the standard deviation of each single shots. Those standard deviation results are plotted in Figure 17 for the three different pressures. It is interesting to notice that a low imposed equivalence ratio 
leads to a relatively low absolute precision whereas the absolute uncertainties tend to increase with equivalence ratio up to 0.7 . Then, one can notice again a decrease in the absolute precision of single shots. One can also notice that an increase in pressure tends to increase the precision of the technique as the absolute uncertainties are the lowest for the highest pressure. If one deals with relative uncertainties, the present technique has $20 \%$ accuracy on the lean side when analyzing single shot data and typical $5 \%$ close to stoichiometric conditions. To determine the spatial distribution of fuel at 5.0 MPa, and discuss possible temporal changes, one would need to take a trade-off between uncertainties and temporal resolution, keeping in mind that each individual measurement is performed within $7 \mu \mathrm{s}$.

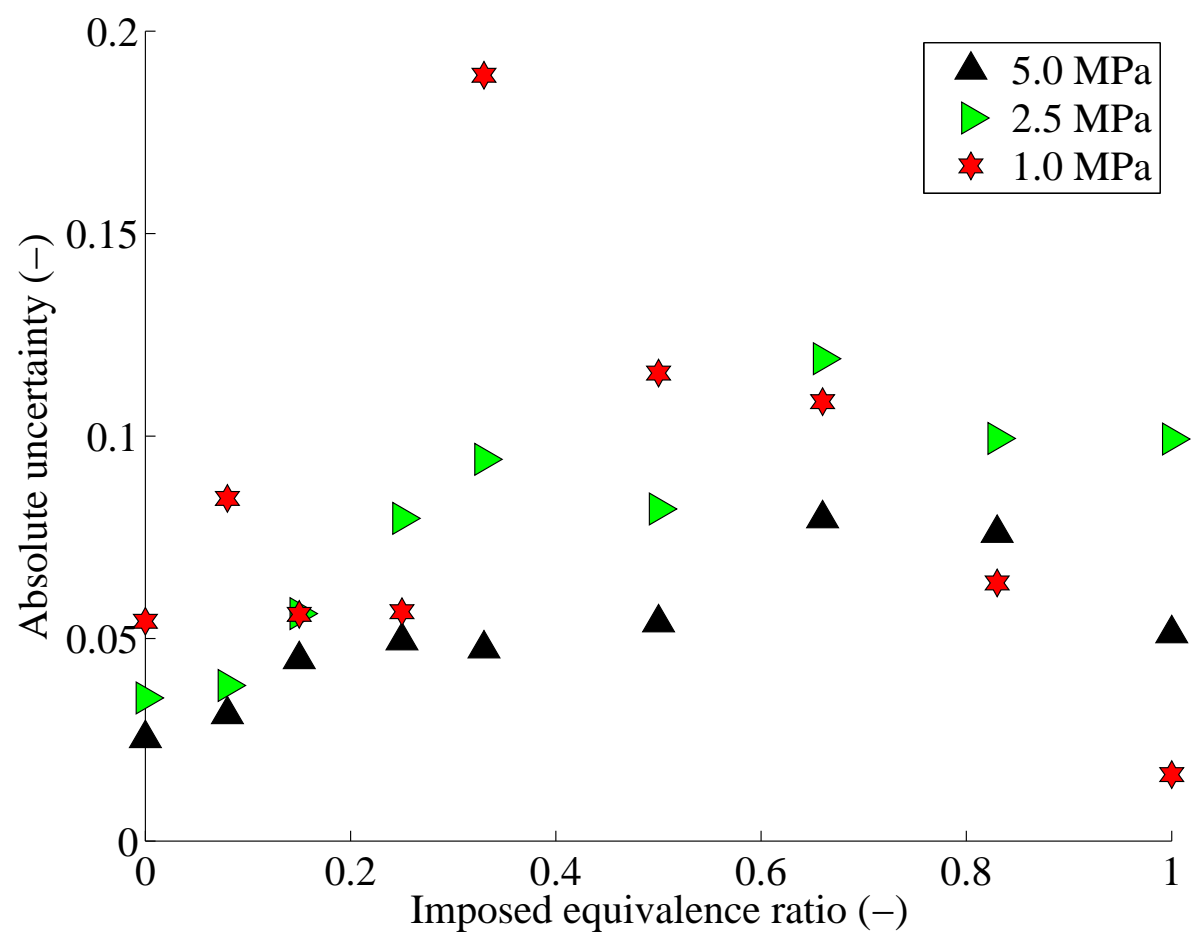

Fig. 17 Single shot uncertainties at different pressures for the ratio $C(833) / N(744)$.

\subsection{Alternative strategies}

Based on the previous results, typical precision is of the order of 0.05 in terms of absolute equivalence ratio measurements. Whereas those values are relatively low when dealing with near stoichiometric conditions, the use of lean conditions lead to a high relative uncertainty. Alternative solution to the peak to peak ratios may be used to overcome this limitation. A possible alternative is to simulate the spectra, including collisional effects and use fitting function to retrieve absolute concentration. This leads 
to some problems as the complete simulation of the physical processes involved in Laser Induced Plasma Spectroscopy may not be straight forward. Another alternative is to compute the correlation between the instantaneous spectra with the experimentally obtained mean data at different equivalence ratio and to use a second order degree polynomial approximation to retrieve the position of the peak. This polynomial fit will then provide the value of the equivalence ratio. This second alternative offers in principle a better efficiency as all the different optical parameters and efficiencies of the collecting system are taken into account.

When applying this procedure to the spectra presented in Figure 15, one obtains a correlation coefficient with respect to the different equivalence ratio used for the constructing the spectral data base. The typical values of the different coefficients are presented in Figure 18 with the database obtained at some discrete values depicted by the circles. The correlation coefficient is 0.973 for the case of the spectra obtained at an equivalence ratio of $0.50,0.944$ at 0.33 and 0.946 at 0.66 . The interpolated value of the equivalence ratio is $0.498 \pm 0.01$, which is in very good agreement with the average spectra for an equivalence ratio of 0.50 . The correlation does not reach a value of unity due to single shot noise.

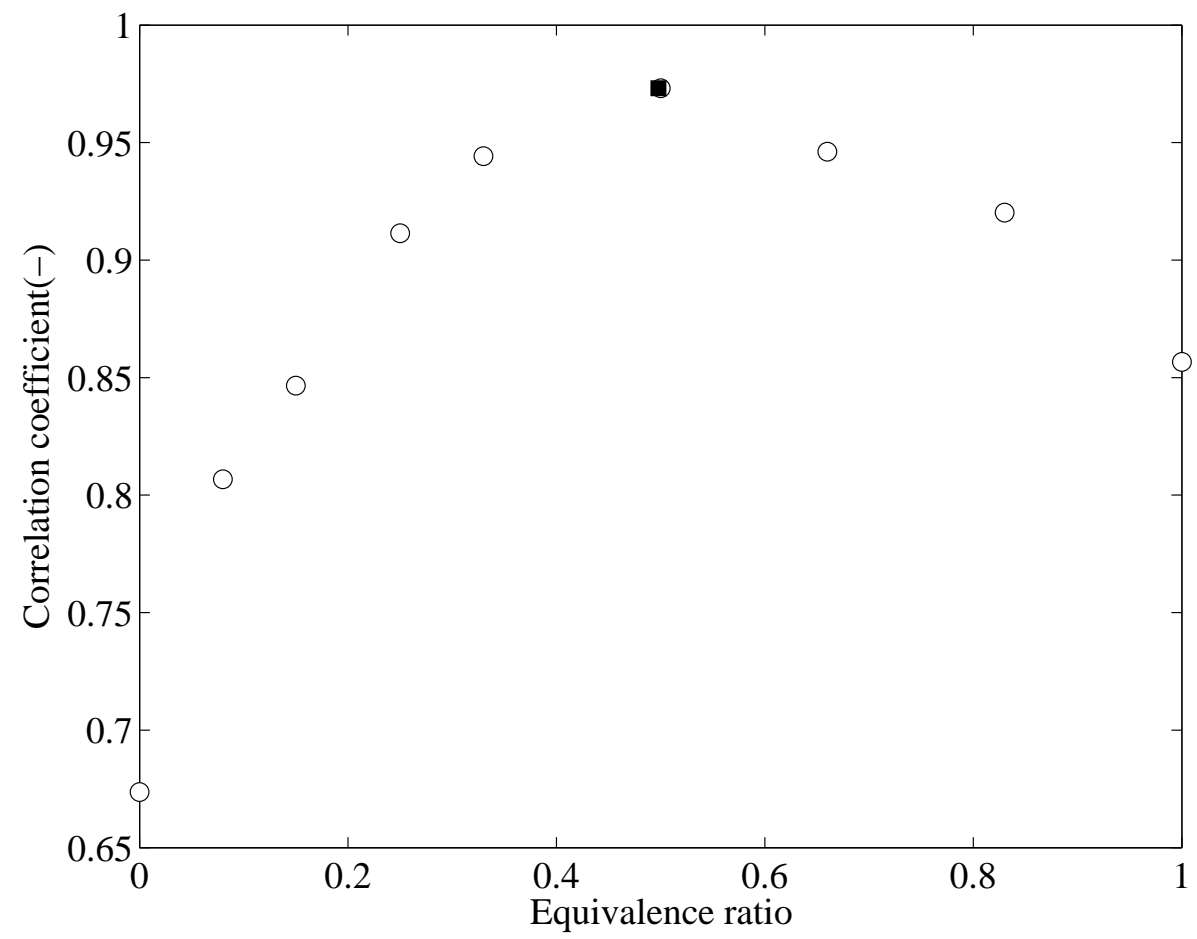

Fig. 18 Evolution of the correlation coefficient as function of the imposed equivalence ratio for a spectra obtained with an imposed equivalence ratio of 0.50 at $5.0 \mathrm{MPa}$

Applying the same correlation procedure to all the database, it is possible to obtain the averaged values but also the standard deviation. Whereas averaged values do 
match with the imposed one, by definition, the values obtained for the standard deviation present interesting lower values as compared to peak ratio analyses. Results are summarized in Figure 19. One can see several interesting features. The first is that the absolute uncertainty remains limited for a pressure of 5.0 MPa as for lean mixture, it only accounts to 0.025 compared to 0.05 when using a peak ratio approach. One can also notice again that an increase in pressure increases the accuracy of the technique when using the carbon emissions around $711 \mathrm{~nm}$ and $833 \mathrm{~nm}$. The accuracy for an intermediate pressure is for some cases half the accuracy at the highest pressure; but this is due to some erroneous data that bias the standard deviation. For clarity, those have not been removed as in actual case, it would be difficult to remove data as the range of equivalence ratio may not be really assessed. Another feature is that the absolute uncertainty increases with imposed equivalence ratio, but the relative uncertainty remains fairly constant around $7.5 \%$ as single shot accuracy at 5.0 MPa. Data obtained at the lowest pressure (1.0 MPa) do not show very consistent tendency and again, as in the peak ratio approach, do not seem to be useful.

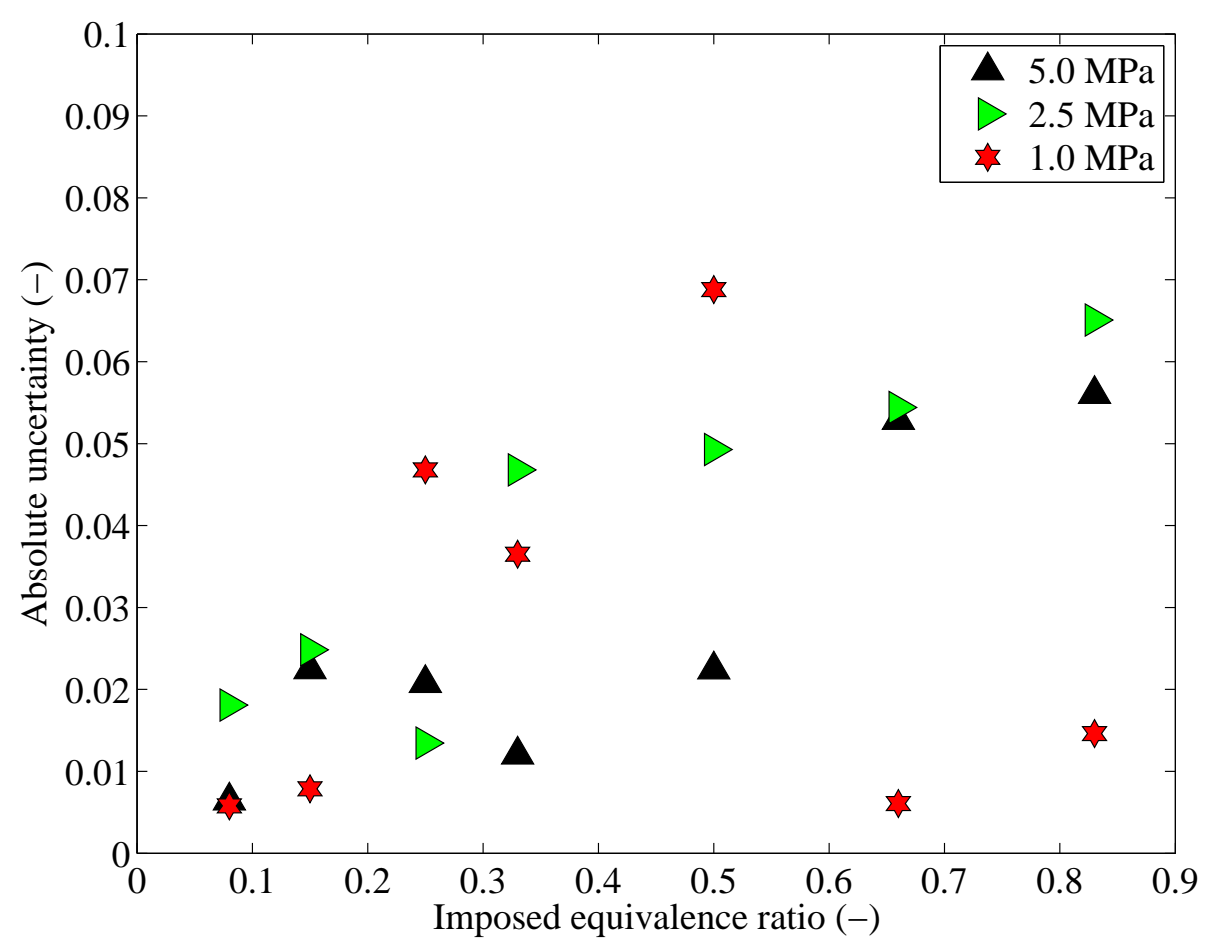

Fig. 19 Evolution of the absolute standard deviation obtained by the spectral fitting procedure as function of imposed equivalence ratio pressures ranging between 1.0 MPa and 5.0 MPa

For lower pressures, an approach based on hydrogen $\mathrm{H}_{\alpha}$ and $\mathrm{H}_{\beta}$ with respect to the triplet of nitrogen around $742-746 \mathrm{~nm}$ and oxygen around $777 \mathrm{~nm}$ remains certainly the best choice as typical uncertainties reported in Zimmer and Tachibana (2007) were less than $4 \%$ for single shot measurements. 


\section{Conclusions}

Laser Induced Plasma Spectroscopy measurements were performed for pressures up to 5.0 MPa. A study on plasma shape showed the influence of carbon in the development of the induced plasma. Its length tended to become longer in presence of carbon which increases the probe volume. Furthermore, several spectroscopic features were determined when adding carbon inside the test cell. A study of their temporal evolution showed a distinctive behavior as compared to typical other atomic emissions. A strategy for measuring mixture composition a $5.0 \mathrm{MPa}$ could be proposed based on the ratio of $\mathrm{C}$ around $833 \mathrm{~nm}$ to the emission of the triplet of $\mathrm{N}(\mathrm{I})$ around $742-746 \mathrm{~nm}$. A good sensitivity was obtained for pressures above $2.5 \mathrm{MPa}$, whereas calibration curves for lower pressures were less sensitive. Using a correlation based calibration clearly improves the precision of the technique and typical single shot uncertainties are limited to $7.5 \%$ at $5.0 \mathrm{MPa}$ when using the spectral information between $700 \mathrm{~nm}$ and $850 \mathrm{~nm}$. This technique has some limitations. The probe volume depends on different parameters and therefore should always be measured. This quite long probe volume may be an issue in cases with strong gradients. One may limit the probe volume by adjusting the laser energy. It requires a relatively long exposure time $(5 \mu \mathrm{s})$ and therefore may only be applicable to cases for which this integration time is smaller than the typical time scales. However, it is easily applied to liquid fuel which is not completely prevaporized. The next steps consist in applying such strategies in real combustor to show spatial and temporal distribution of kerosene in actual gas turbine environment as LIPS technique is not sensitive to molecular content of kerosene (Zimmer and Yoshida (2008)) or initial temperature. This technique would also work for richer mixtures for which carbon related peaks have stronger emissions. It was not investigated in the present studies because the focus was put on the lean side only.

\section{Acknowledgments}

The first author wishes to acknowledge the financial support of the CNRS for providing the grants to travel through the Programme International de Collaboration Scientifique, number 4979 .

\section{References}

S. Acquaviva. Simulation of emission molecular spectra by a semi-automatic programme package: the case of $\mathrm{c} 2$ and $\mathrm{cn}$ diatomic molecules emitting during laser ablation of a graphite target in nitrogen environment. Spectrochimica Acta Part A: Molecular and Biomolecular Spectroscopy, 60(8-9):2079-2086, 72004.

Z.A. Arp, D.A. Cremers, R.D. Harris, D.M. Oschwald, G.R. Parker, and D.M. Wayne. Feasibility of generating a useful laser-induced breakdown spectroscopy plasma on rocks at high pressure: preliminary study for a venus mission. Spectrochimica Acta Part B: Atomic Spectroscopy, 59(7):987-999, 2004.

D. Bradley, C. G. W. Sheppard, I. M. Suardjaja, and R. Woolley. Fundamentals of high-energy spark ignition with lasers. Combustion and Flame, 138(1-2):55-77, 2004. 
R. Devillers, G. Bruneaux, and C. Schulz. Investigation of toluene lif at high pressure and high temperature in an optical engine. Applied Physics B: Lasers and Optics, 96(4):735-739, 2009.

F. Ferioli and S.G. Buckley. Measurements of hydrocarbons using laser-induced breakdown spectroscopy. Combustion and Flame, 144(3):435-447, 2006.

R. K. Hanson. Applications of quantitative laser sensors to kinetics, propulsion and practical combustion system. Proceedings of the Combustion Institute, 33:1-40, 2011.

J. Kojima and Q.-V. Nguyen. Measurement and simulation of spontaneous raman scattering in high-pressure fuel-rich h2-air flames. Measurement Science and Technology, 15(3):565-580, 2004.

M. Orain, F. Grisch, E. Jourdanneau, B. Rossow, C. Guin, and B. Trétout. Simultaneous measurements of equivalence ratio and flame structure in multipoint injectors using plif. Comptes Rendus Mécanique, 337(6-7):373-384, 2009.

T.X. Phuoc and F.P. White. Laser-induced spark for measurements of the fuel-to-air ratio of a combustible mixture. Fuel, 81(13):1761-1765, 2002.

R.W. Schmieder. Combustion applications of laser induced breakdown spectroscopy. Technical Report Report No. SAND81-8886, Sandia National Laboratories, Livermore, Calif., 1981.

C. Schulz and V. Sick. Tracer-lif diagnostics: quantitative measurement of fuel concentration, temperature and fuel/air ratio in practical combustion systems. Progress in Energy and Combustion Science, 31(1):75-121, 2005.

P. Stavropoulos, A. Michalakou, G. Skevis, and S. Couris. Quantitative local equivalence ratio determination in laminar premixed methane-air flames by laser induced breakdown spectroscopy (libs). Chemical Physics Letters, 404(4-6):309-314, 2005.

V. Sturm and R. Noll. Laser-induced breakdown spectroscopy of gas mixtures of air, co2, n2, and c3h8 for simultaneous c, h, o, and n measurement. Appl. Opt., 42(30): 6221-6225, 2003.

E. Vors, C. Gallou, and L. Salmon. Laser-induced breakdown spectroscopy of carbon in helium and nitrogen at high pressure. Spectrochimica Acta Part B: Atomic Spectroscopy, 63(10):1198 - 1204, 2008.

L. Zimmer and S. Tachibana. Laser induced plasma spectroscopy for local equivalence ratio measurements in an oscillating combustion environment. Proceedings of the Combustion Institute, 31(1):737-745, 2007.

L. Zimmer and S. Yoshida. Measurements of mixture fraction with laser induced plasma spectroscopy. In Journal of the Combustion Society of Japan, volume 50, pages 317-324, 2008.

L. Zimmer, K. Okai, and Y. Kurosawa. Combined laser induced ignition and plasma spectroscopy: Fundamentals and application to a hydrogen-air combustor. Spectrochimica Acta Part B: Atomic Spectroscopy, 62:1484-1495, 2007. 